\title{
Bewertung von Kapitalgesellschaften: Der Steuervorteil der Fremdfinanzierung unter schweizerischem Steuerrecht
}
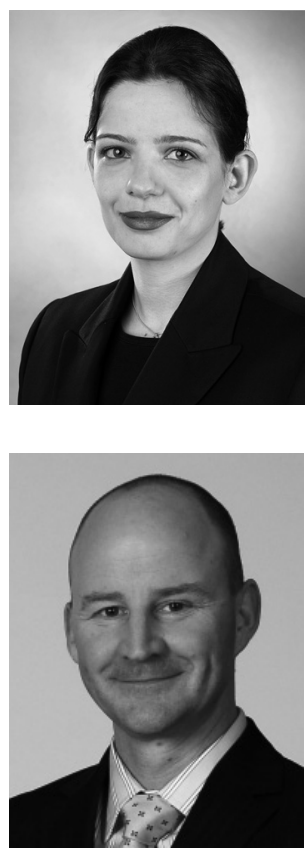

\section{Carmen Bachmann und Wolfgang Schultze}

Unternehmensbewertung, Tax Shield, Schweiz, Fremdfinanzierung, Steuersystem, Discounted Cashflow-Methodik

Company valuation, Switzerland, debt financing, tax system, discounted cash flow method

Die Ermittlung des steuerlichen Wertvorteils aus der Fremdfinanzierung einer Unternehmung spielt eine zentrale Rolle bei der Unternehmensbewertung. Dabei wird regelmässig eine typisierende Steuerbelastung zugrunde gelegt. Nicht selten weichen die tatsächlich eintretenden Steuerfolgen hiervon signifikant ab. Dies gilt umso mehr, wenn ein schweizerisches Unternehmen bewertet wird. Den steuerlichen Besonderheiten der einzelnen Kantone kann eine pauschalisierende Steuerberechnung unmöglich gerecht werden. Dies führt zu teils erheblichen Ungenauigkeiten bei der Bewertung von Unternehmen. Daher berücksichtigt der vorliegende Beitrag die Besonderheiten des schweizerischen Steuerrechts und integriert diese in die Bewertungsformeln der Discounted Cashflow-Methodik. Es zeigt sich, dass das Tax Shield in Abhängigkeit von der Ausgestaltung der jeweiligen kantonalen Steuerrechtsordnungen stark variiert und teilweise sogar negativ werden kann.

The calculation of the tax benefit of debt financing plays an essential role in the valuation of companies. Often, a simplified average tax rate is utilized. The actual tax consequences, however, often differ significantly. This holds even more true for the valuation of Swiss companies. A generalizing tax calculation inevitably falls short of the peculiarities of the respective cantons. This may result in material valuation inaccuracies. As a consequence, this article appreciates the particularities of the Swiss tax system and integrates them in the valuation formulas. It appears that the tax shield may vary strongly or may even become a negative value depending on the applicable cantonal tax system.

\section{Einleitung}

Der Steuervorteil der Fremdfinanzierung ist ein wesentlicher Bestandteil der Unternehmensbewertung. Bei der Ermittlung dieses Steuervorteils wurde bisher regelmässig ein typisierendes Steuersystem zugrunde gelegt (vgl. stellvertretend Brealey et al. 2008, 530ff.). Jüngere Untersuchungen haben nachgewiesen, dass sich hieraus zum Teil erhebliche Bewertungsungenauigkeiten ergeben können und eine zutreffende Wertermittlung nur bei Berücksichti- 
gung der jeweils einschlägigen Steuersysteme gelingen kann. ${ }^{1}$ Dies ist ein massgeblicher Erkenntnisgewinn für die Steuerwirkungen bei der Unternehmensbewertung, wurde bisher jedoch lediglich im Zusammenhang mit deutschen Gesellschaften untersucht.

Für eine schweizerische Kapitalgesellschaft wurden diese Einflüsse trotz der erheblichen theoretischen und praktischen Relevanz noch nicht analysiert. Dies erscheint jedoch zwingend erforderlich, da sich aufgrund des föderalen Charakters des Steuersystems und dem damit verbundenen „Steuergefälle“ selbst im rein nationalen Fall erhebliche Unterschiede ergeben können. Neben der direkten Bundessteuer müssen die Steuergesetze der 26 Kantone beachtet werden, die ihrerseits teilweise grundlegend voneinander abweichen. Hinzu kommt vereinzelt noch eine Besteuerung durch die ca. 2800 Gemeinden.

Ist das Bewertungsobjekt eine Kapitalgesellschaft, so ist die effektive Steuerbelastung unter Berücksichtigung der beiden Ebenen Gesellschaft und Gesellschafter zu ermitteln. Ist der Gesellschafter in einem anderen Kanton als die Gesellschaft ansässig oder sind mehrere Gesellschafter in unterschiedlichen Kantonen steuerpflichtig ( „Hochsteuer-“ bzw. „Niedrigsteuer-Kantone“), kann sich hieraus eine höchst unterschiedliche effektive Steuerbelastung ergeben. Ferner ist der Einfluss des derzeitigen Körperschaftsteuersystems zu berücksichtigen. Seit 1.1.2009 wird - anders als bisher - die Doppelbesteuerung von Unternehmensgewinnen auf Gesellschafts- und Gesellschafter-Ebene abgemildert (Unternehmenssteuerreform II). Dividenden unterliegen beim Gesellschafter nunmehr nur noch einer teilweisen Besteuerung, um die Vorbelastung mit Körperschaftsteuer (Ausschüttung aus dem Ergebnis der Kapitalgesellschaft nach Steuern) zu kompensieren. Dies gilt unmittelbar für die Bundessteuer. Ein Großteil der Kantone folgt diesem Modell.

Um die schweizerischen Besonderheiten bei der Ermittlung des Tax Shields herauszuarbeiten, ist der Beitrag wie folgt strukturiert: Angenommen wird, dass einer schweizerischen Kapitalgesellschaft Finanzmittel von einem Gesellschafter oder von einem fremden Dritten zugeführt werden. Zunächst wird die Besteuerungssituation der Gesellschaft bei Eigenfinanzierung bzw. bei Fremdkapitalaufnahme dargestellt. Sodann wird auf Ebene des Kapitalgebers danach differenziert, ob die Mittelvergabe aus Privat- oder Geschäftsvermögen erfolgt. Für diese Fallkonstellationen werden die Besteuerungsregeln bis zur Ebene des letzten Kapitalgebers aufgezeigt, um daraus das Tax Shield und damit Aussagen über die steuerliche Vorteilhaftigkeit der Fremdfinanzierung abzuleiten. Im Anschluss werden daraus die relevanten Bewertungsgleichungen für die Discounted Cashflow-Methodik entwickelt. Hieraus ergibt sich, dass die jeweils anwendbaren kantonalen Steuerrechtsordnungen zwingend zu berücksichtigen sind, um zu sinnvollen und realitätsgetreuen Unternehmenswerten zu gelangen.

\section{Annahmen und Fallkonstellation}

Zur Berechnung des Tax Shields (TS) ist der Unterschied zwischen dem Gesamteinkommen der Investoren eines verschuldeten und eines identischen unverschuldeten Unternehmens zu ermitteln.

1 Vgl. u.a. Schultze (2003, 546ff.), Ballwieser et al. (2007, 765), Bachmann/Schultze (2008, 9ff.). Mit der Einführung des Halbeinkünfteverfahrens in Deutschland ist eine grosse Zahl von Beiträgen erschienen, die eine Neuberechnung der Höhe des Steuervorteils aus der Fremdfinanzierung, dem sog. Tax Shield zum Gegenstand hatte. Vgl. insbesondere Auge-Dickhut et al. (2000), Schüler (2000), Baetge et al. (2001), Lobe (2001), Dinstubl (2002), Drukarczyk/Lobe (2002a, b), Husmann et al. (2002a, b), Drukarczyk (2003), Schultze (2005). Diese Beiträge sind jedoch auf das deutsche Steuerrecht beschränkt. 
Es werden hierbei nachfolgende Annahmen getroffen:

1) Es werden ausschliesslich Gewinne erzielt.

2) Da die jährliche Steuerbelastung eine Funktion der Geschäftsergebnisse früherer Jahre ist, sei der Reingewinn konstant (siehe ebenso die Annahmen des Bundesamtes für Statistik, in: ESTV 2008, 46).

3) Es wird von einer Vollausschüttung der Gewinne ausgegangen. Damit wird ein - im Falle einer residualen Ausschüttung entstehender - Tilgungsdifferenzeffekt ausser Acht gelassen. ${ }^{2}$

4) Es wird ein vollkommener Kapitalmarkt unterstellt, sodass der Preis der Alternativinvestition hinreichend bestimmt und somit durch Diskontierung auf das Bewertungsobjekt übertragbar ist.

5) Unternehmen treffen Investitions- und Finanzierungsentscheidungen unabhängig voneinander.

6) Bewertungsobjekt ist eine Handels-, Industrie- oder Bank-AG ohne Beteiligungen.

7) Die Kantone folgen bei der Besteuerung von Dividenden dem Modell des Bundes und besteuern diese wie auf Bundesebene entsprechend der Unternehmenssteuerreform II.

8) Das Einkommen natürlicher Personen unterliegt auf Bundesebene dem Grenzsteuersatz von $11,5 \%$ (Art. 214 DBG).

9) Die Belastung mit Kapitalsteuer, welche nur auf kantonaler bzw. kommunaler Ebene erhoben wird ${ }^{3}$, wird im Folgenden zwar dargestellt, jedoch hinsichtlich der Belastung weitgehend ausser Acht gelassen. Dies ist insbesondere aufgrund der Unternehmenssteuerreform II vertretbar, da in einigen Kantonen die Kapitalsteuer um den Betrag der Gewinnsteuer reduziert werden soll, d.h. die geschuldete Gewinnsteuer kann von der Kapitalsteuer abgezogen werden (SSK 2009, 3). Sobald die geschuldete Gewinnsteuer grösser ist als die geschuldete Kapitalsteuer, fällt die gesamte Belastung mit Kapitalsteuer weg. Darüber hinaus finden sich bei Beteiligungs- und Holdingsgesellschaften ohnehin umfangreiche Erleichterungen bezüglich der Kapitalsteuer.

10) Die Vermögensteuer der natürlichen Personen, welche von den Kantonen und Gemeinden erhoben wird, bleibt ausser Ansatz. Hierbei handelt es sich nur um eine Ergänzungssteuer, d. h. Steuermass und Bewertungsregeln sind entsprechend zurückhaltend ausgestaltet (ausführlich in SSK 2008b, 1ff.)

11) Die Eignerkapitalgesellschaft ist eine Beteiligungsgesellschaft mit einer mindestens $20 \%$ igen Beteiligung an der Bewertungskapitalgesellschaft. Die Voraussetzung für den sog. Beteiligungsabzug (95\% Dividendenfreistellung, ausführlich unter 3.1.3.2.1) ist damit erfüllt. Wird diese Beteiligungsgrenze unterschritten, kommt eine Vergünstigung (sog. Holdingprivileg) nur dann in Betracht, wenn die Eignerkapitalgesellschaft die Kriterien einer Holdinggesellschaft erfüllt (ausführlich unter 3.1.3.2.2). In beiden Fällen

2 Weicht die Höhe des Gewinns von der der Ausschüttungen ab, ergeben sich Veränderungen der Gewinnrücklagen, die wiederum zu einer temporär unterschiedlichen Steuerbelastung der Investoren führen, so dass ein Steuerstundungseffekt ausgelöst wird, der zusätzlich zum hier betrachteten Zins-Tax Shield zu berücksichtigen ist (Schultze 2005, 253). Zur kontroversen Diskussion des Tilgungseffekts siehe Schüler (2000, 153ff.), Baetge et al. (2001, 318), Dinstubl (2002, 83), Husmann et al. (2002a, 33), Laitenberger (2002, 555ff.) und Laitenberger $(2003,1226)$.

3 Während der Bund nur den Gewinn besteuert, erheben die Kantone darüber hinaus auch eine Steuer auf das Kapital. Steuerobjekt ist das Eigenkapital. Gem. Art. 29 Abs. 2 StHG besteht das steuerbare Eigenkapital aus dem einbezahlten Grund- oder Stammkapital, den offenen und den aus versteuertem Gewinn gebildeten stillen Reserven. Der Steuersatz ist proportional ausgestaltet und bewegt sich zwischen 0,3 und 7 \%. 
werden die Dividendeneinnahmen auf Ebene des Anteilseigners von der Besteuerung weitgehend ausgenommen.

Es wird von folgender Fallkonstellation ausgegangen: Die zu bewertende Kapitalgesellschaft ist ansässig im Kanton A. Das zu ihrer Finanzierung erforderliche Kapital kann von den Kapitalgebern alternativ als Eigen- oder Fremdkapital bereitgestellt werden. Bei den Kapitalgebern kann es sich um natürliche oder juristische Personen handeln, wobei wir uns im letzteren Fall auf die Rechtsform Kapitalgesellschaft beschränken. Eigen- und Fremdkapitalgeber sowie das Bewertungsobjekt können zudem ihren Sitz in verschiedenen Kantonen (A, B, C) haben. Diese verschiedenen Alternativen werden in Abbildung 1 veranschaulicht. Diese Alternativen enthalten auch die Möglichkeit der mehrheitlichen Eigenfinanzierung durch eine Kapitalgesellschaft (Mutterkapitalgesellschaft), was wir als Spezialfall untersuchen. Denkbar ist auch, dass die Eignerkapitalgesellschaft selbst der Bewertungsgesellschaft Fremdkapital zur Verfügung stellt, dass es sich also bei Eigner- und Gläubigerkapitalgesellschaft um ein und dieselbe Gesellschaft handelt (Gesellschafterfremdfinanzierung).

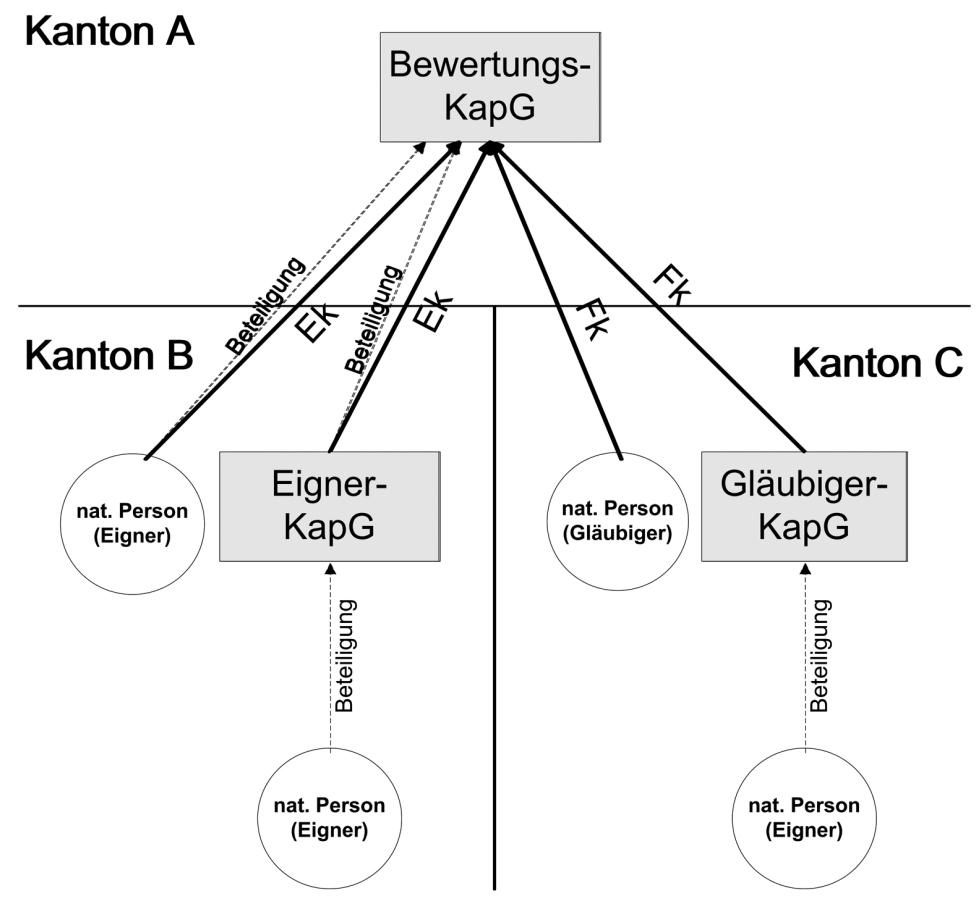

Abbildung 1: Fallkonstellation

Der Eigenfinanzierung durch eine Eignerkapitalgesellschaft ist hinsichtlich der Steuerbelastung grundsätzlich auch die Konstellation einer Aufteilung auf mehrere Eignerkapitalgesellschaften, die jeweils zu mindestens $20 \%$ an der Bewertungskapitalgesellschaft beteiligt sind, gleichgestellt. Lediglich dann, wenn eine dieser Eignerkapitalgesellschaften die für den Beteiligungsabzug relevante Beteiligungsgrenze von $20 \%$ unterschreitet, kommt für diese Eignergesellschaft kein Beteiligungsabzug mehr in Betracht. Soweit sie die Anteilsrechte nur in geringerem Umfang in ihrem Beteiligungsportefeuille hält, ist zu prüfen, ob sie die Voraus- 
setzungen einer Holdinggesellschaft erfüllt (siehe 3.1.3.2.2). Entsprechend den oben getroffenen Annahmen wird hier davon ausgegangen, dass eine Beteiligungsgesellschaft mit Erfüllen der Voraussetzungen des Beteiligungsabzugs vorliegt. Eine Gruppenbesteuerung verflochtener Gesellschaften kennt das schweizerische Steuerrecht nicht, so dass sich der steuerliche Belastungsunterschied zwischen Mehrheits- und Minderheitsaktionären auf die Anwendung des Beteiligungsabzugs bzw. des Holdingprivilegs beschränkt.

$\mathrm{Da}$ die Besteuerungsregelungen im Kanton A identisch mit denen vom Kanton B sein können, kann implizit auch der Fall erfasst werden, dass sich die gesamte Struktur in ein und demselben Kanton befindet. Ebenso kann durch Annahme einheitlicher Steuerfolgen im Kanton A und C die Konstellation, dass Fremdkapitalgeber und -nehmer dem gleichen Kanton zuzuordnen sind, erfasst werden. Denkbar ist auch, dass sich alles innerhalb eines Kantons abspielt.

\section{Die Steuerbelastung der Einkünfte aus der Kapitalgesellschaft}

\subsection{Steuerliche Belastung von Eigenkapitalgeber-Einkünften}

Grundsätzlich erfolgt eine Besteuerung von Kapitalgesellschaften auf zwei Ebenen: Neben der Belastung mit Körperschaftsteuer auf Ebene der Gesellschaft kommt es im Ausschüttungsfall zu einer Besteuerung auf Ebene des Anteilseigners. Bei der Ausschüttung wird gem. Art. 13 S. 2 VStG eine Verrechnungssteuer i. H. v. 35\% erhoben, welche jedoch auf die tatsächlich geschuldete Steuer angerechnet werden kann. Da es sich daher nur um eine besondere Erhebungsform der Steuer handelt, die letztendlich keinen Einfluss auf die Höhe der tatsächlichen Steuerlast hat, wird die Verrechnungssteuer im Folgenden ausser Acht gelassen.

\subsubsection{Besteuerung der zu bewertenden Kapitalgesellschaft}

Die schweizerische Kapitalgesellschaft unterliegt sowohl der Besteuerung durch die direkte Bundessteuer ${ }^{4}$ als auch der durch die kantonalen Steuern. ${ }^{5}$ Hinzu kommt häufig eine kommunale Steuerbelastung durch die Gemeinden.

Die Kapitalgesellschaft ist als selbstständiges Steuersubjekt nach dem Art. 50 DBG i. V. m. Art. 52 DBG und Art. 20 StHG unbeschränkt steuerpflichtig, wenn sich ihr Sitz oder ihre tatsächliche Verwaltung in der Schweiz befindet. Nahezu gleichlautend ist die Definition der unbeschränkten Steuerpflicht in den kantonalen Steuergesetzen, dort jedoch mit der Beschränkung auf das jeweilige Kantonsgebiet.

Gem. Art. 68 DBG beträgt die Gewinnsteuer der Kapitalgesellschaften auf Bundesebene proportional 8,5\%. Bemessungsgrundlage ist der nach Art. 58 DBG zu ermittelnde steuerbare Reingewinn. Dieser ergibt sich aus dem Saldo der Erfolgsrechnung abzüglich der geschäftsmässig nicht begründeten Kosten und Aufwendungen. Hervorzuheben ist, dass gem. Art. 59 S. 1 Buchst. a) DBG die eidgenössischen, kantonalen und kommunalen Steuern zum geschäftsmässig begründeten Aufwand zählen. Dies bedeutet auch, dass eine sehr hohe Kantonssteuer zu einer geringeren Bundessteuerbelastung führt, da die Kantonssteuer die Be-

4 Rechtsgrundlage ist das Bundesgesetz über die direkte Bundessteuer (DBG) vom 14.12.1990 (Stand: 1.1.2009).

5 Rechtsgrundlage bilden die jeweiligen kantonalen Steuergesetze und das Bundesgesetz über die Harmonisierung der direkten Steuern der Kantone und Gemeinden (StHG) vom 14. 12.1990 (Stand: 1.1.2009). 
messungsgrundlage der Bundessteuer mindert. Mithin tritt durch die Abzugsfähigkeit der Steuerbelastung von ihrer eigenen Grundlage ein gewisser Kompensationseffekt ein.

Auf kantonaler Ebene kann der Tarif im Rahmen der Gewinnsteuer proportional ${ }^{6}$, gemischt $^{7}$ oder progressiv $^{8}$ ausgestaltet sein (ausführlich SSK 2008a, 22). Bemessungsgrundlage ist hier ebenfalls der Reingewinn, dessen Definition in Art. 24 StHG in Anlehnung an die des DBG erfolgt. Auch auf kantonaler Ebene gehören Steuern zum geschäftsmässigen Aufwand (Art. 25 S. 1 Buchst. A StHG). Neben den Kantonen erheben auch die Gemeinden Steuern, soweit sie hierzu durch den übergeordneten Kanton ermächtigt sind. Regelmässig erfolgt dies in Abhängigkeit vom kantonalen Steuertarif (SSK 2008a, 68f.). Der effektive Steuersatz auf Kantons- und Gemeindeebene kann je nach Kanton und Gemeinde sehr stark variieren.

Da die Steuern vom Reingewinn abgezogen werden dürfen (Abzugsgrössen von sich selbst) kann die Steuer nicht einfach durch Multiplikation von Bemessungsgrundlage und Steuersatz ermittelt werden, sondern ist auch die innere Abzugsfähigkeit der Steuer zu berücksichtigen.

Der Effektivsteuersatz für die Gesamtsteuerbelastung in A lautet damit:

$\mathrm{s}_{\mathrm{Kap}, \mathrm{A}}=\frac{\mathrm{s}_{\mathrm{Kap}}^{\mathrm{BSt}}+\mathrm{s}_{\mathrm{Kap}, \mathrm{A}}^{\mathrm{kkSt}}}{1+\mathrm{s}_{\mathrm{Kap}}^{\mathrm{BSt}}+\mathrm{s}_{\mathrm{Kap}, \mathrm{A}}^{\mathrm{kkt}}}=\frac{0,085+\mathrm{s}_{\mathrm{Kap}, \mathrm{A}}^{\mathrm{kkSt}}}{1,085+\mathrm{s}_{\mathrm{Kap}, \mathrm{A}}^{\mathrm{kkSt}}}$

wobei:

$\mathbf{S}_{\text {Kap,A }} \quad$ Effektivsteuersatz der Kapitalgesellschaft im Kanton A

$\mathrm{S}_{\mathrm{Kap}}^{\mathrm{BSt}} \quad$ Bundessteuersatz für Kapitalgesellschaften (proportional 8,5\%)

$\mathrm{S}_{\mathrm{Kap}, \mathrm{A}}^{\mathrm{kkSt}} \quad$ Steuersatz für Kapitalgesellschaften auf kantonaler/kommunaler Ebene im Kanton A

Bei einem steuerbaren Kapital von 100.000 CHF ergeben sich - abgeleitet aus den Tabellen des Statistischen Bundesamtes von 2008 (ESTV 2008, 48ff.) - beispielsweise mindestens bzw. höchstens die in Abbildung 2 und Abbildung 3 aufgeführten Effektivsteuersätze und Mittelwerte (kantonale/kommunale Steuern ohne direkte Bundessteuer) für Gewinn- und Kapitalsteuern in Abhängigkeit vom Reingewinn:

\begin{tabular}{|l|l|l|l|c|}
\hline Reingewinn & $4.000 \mathrm{CHF}$ & $8.000 \mathrm{CHF}$ & $20.000 \mathrm{CHF}$ & $30.000 \mathrm{CHF}$ \\
\hline mindestens & ca. $8 \%($ Zug) & ca. $7 \%$ (Zug) & ca. $7 \%$ (Sarnen) & ca. 6\% (Zug) \\
\hline maximal & ca. $38 \%($ Genf) & ca. $30 \%$ (Genf) & ca. $28 \%$ (Basel) & ca. $25 \%$ (Genf) \\
\hline Mittelwert & ca. $21 \%$ & ca. $16 \%$ & ca. $14 \%$ & ca. $14 \%$ \\
\hline
\end{tabular}

Abbildung 2: Effektivsteuersätze bei einem steuerbaren Kapital von $100.000 \mathrm{CHF}$

6 Bei dem proportionalen Tarif wird ein einziger fester proportionaler Tarif verwendet, welcher jedoch in einzelnen Kantonen erst ab einer bestimmten Gewinnhöhe beginnt und bis zu diesem Schwellenwert ausgestaltet ist.

7 Im Fall sog. gemischter Systeme (Zwei- oder Dreistufentarif) wird differenziert nach (i) verschiedenen Proportionalzonen in Abhängigkeit vom Gewinn, d.h. es ergibt sich eine leichte Progression, (ii) der Ertragsintensität, d.h. es wird eine gewisse Grundsteuer erhoben, die durch einen Zuschlag in Abhängigkeit von der Ertragsintensität erhöht wird oder (iii) sowohl nach Gewinnhöhe als auch nach Ertragsintensität.

8 Der progressive Steuertarif ist teilweise so ausgestaltet, dass er sich nach der Höhe des Gewinns bestimmt, teilweise erfolgt eine Orientierung an der Ertragsintensität. 
Bei einem steuerbaren Kapital von 2.000.000 CHF beträgt der Mindest- bzw. HöchstEffektivsteuersatz:

\begin{tabular}{|l|l|l|l|l|}
\hline Reingewinn & $80.000 \mathrm{CHF}$ & $160.000 \mathrm{CHF}$ & $400.000 \mathrm{CHF}$ & $600.000 \mathrm{CHF}$ \\
\hline mindestens & ca. $8 \%$ (Zug) & ca. $7 \%$ (Herisau) & ca. $6 \%$ (Herisau) & ca. $6 \%$ (Herisau) \\
\hline maximal & ca. $38 \%$ (Genf) & ca. $30 \%$ (Genf) & ca. $27 \%$ (Basel) & ca. $25 \%$ (Genf) \\
\hline Mittelwert & ca. $22 \%$ & ca. $18 \%$ & ca. $17 \%$ & ca. $16 \%$ \\
\hline
\end{tabular}

Abbildung 3: Effektivsteuersätze bei einem steuerbaren Kapital von 2.000.000 CHF

Die Abnahme der Steuersätze mit zunehmendem Reingewinn in Abbildung 2 und 3 ist auf die Kapitalsteuer zurückzuführen, die bei einer höheren Gewinnsteuerbelastung prozentual weniger ins Gewicht fällt. Da sich die Statistiken auf den Stand vor der Unternehmenssteuerreform II beziehen, wurde hierbei noch nicht berücksichtigt, dass die Kantone künftig die geschuldete Gewinnsteuer von der Kapitalsteuer abziehen bzw. abziehen werden (siehe auch Annahme 9). Es ist jedoch anzunehmen, dass die Möglichkeit der Anrechnung von Kapitalsteuer mit einer Erhöhung der Steuersätze kompensiert wird. Die Spannbreite des effektiven Steuersatzes auf Ebene der Kapitalgesellschaft (kommunale bzw. kantonale Ebene) bewegt sich zwischen Werten von ca. 6\% und ca. 38\%. Der Mittelwert liegt bei ca. $15 \%$.

\subsubsection{Steuerliche Behandlung von Zinsaufwendungen}

Wird der Finanzmittelbedarf der Bewertungskapitalgesellschaft durch die Gewährung eines Darlehens gedeckt, mindern die Zinsaufwendungen ihr steuerpflichtiges Einkommen, so dass auf Ebene der Kapitalgesellschaft eine geringere Belastung an Körperschaftsteuer entsteht. Schuldzinsen zählen grundsätzlich zum geschäftsmässig begründeten Aufwand. Allerdings kann die Abzugsfähigkeit von Zinsaufwendungen im Rahmen der Gesellschafterfremdfinanzierung eingeschränkt sein. Liegt sog. „verdecktes Eigenkapital“ vor, d.h. Fremdkapital, welchem wirtschaftlich die Bedeutung von Eigenkapital zukommt, gehören gem. Art. 65 DBG die Schuldzinsen, die auf jenen Teil des Fremdkapitals entfallen, zum steuerbaren Gewinn (Blumenstein/Locher 2002, 195, m. w. N. in Fn. 61).

In allen Kantonen wird ebenfalls das steuerbare Eigenkapital um den Teil des Fremdkapitals erhöht, dem wirtschaftlich die Bedeutung von Eigenkapital zukommt (Art. 29a StHG). Bislang war die Umqualifizierung in Eigenkapital auch hinsichtlich der Kapitalsteuer bedeutsam, da das Fremdkapital von der Kapitalsteuer nicht erfasst wird. Steuerobjekt ist ausschliesslich das Eigenkapital. Lediglich im Fall einer Unterkapitalisierung wird das steuerbare Eigenkapital um den Teil des Fremdkapitals erhöht, dem wirtschaftlich die Bedeutung von Eigenkapital zukommt (Art. 29a StHG). Wegen der nach der Unternehmenssteuerreform II bestehenden Anrechnungsmöglichkeit der Gewinnsteuer auf die Kapitalsteuer fällt dies jedoch nunmehr nicht ins Gewicht. Dem Kapital kommt insbesondere dann wirtschaftlich die Bedeutung von Eigenkapital zu, wenn eine Gesellschafterfremdfinanzierung vorliegt. Da vorliegend jedoch nicht zwingend von einer Mittelhingabe durch Gesellschafter ausgegangen wird, wird diese Unterkapitalisierungsregelung nicht ins Kalkül mit einbezogen.

\subsubsection{Besteuerung der Anteilseigner}

Schüttet die zu bewertende Kapitalgesellschaft ihren Gewinn aus, richtet sich die Besteuerung auf Ebene der Anteilseigner danach, ob (i) es sich um einen privaten Anteil handelt oder (ii) die Beteiligung im Geschäftsvermögen einer natürlichen Person gehalten wird oder (iii) dieser Anteilseigner eine Kapitalgesellschaft ist. 


\subsubsection{Natürliche Person als Anteilseigner}

Natürliche Personen mit steuerrechtlichem Wohnsitz oder Aufenthalt in der Schweiz sind gem. Art. 3 DBG (bzw. Art. 3 StHG) mit ihrem gesamten Einkommen unbeschränkt steuerpflichtig. Dividenden zählen gem. Art. 20 Abs. 1 Buchst. c DBG zu den Einkünften aus beweglichem Vermögen. Vor dem 1.1.2009 unterlagen diese vollumfänglich der direkten Bundessteuer. Mit Umsetzung der Unternehmenssteuerreform II gilt dies jedoch nur noch für Beteiligungen unter 10\% (Streubesitz, unabhängig davon, ob die Beteiligung Privat- oder Geschäftsvermögen darstellt). Sobald die Beteiligung mindestens $10 \%$ beträgt, unterliegen die Dividenden bei Beteiligungen im Privatvermögen nur noch zu 60\% (Art. 20 Abs. 1bis DBG) und bei Beteiligung im Geschäftsvermögen nur noch zu 50\% der Besteuerung (Art. 18b Abs. 1 DBG). Die (anteilig steuerpflichtigen) Dividenden unterliegen der Einkommensteuer (Grenzsteuersatz 11,5\%).

Entsprechend der oben getroffenen Annahme wird davon ausgegangen, dass die Kantone dieser Freistellung auf Bundesebene folgen, d.h. die Bemessungsgrundlage der Steuern beträgt entweder 100\% (Streubesitz), 60\% (Privatvermögen) oder 50\% (Geschäftsvermögen). ${ }^{9}$

Die effektiven Steuersätze $\mathrm{s}_{\mathrm{ESt}, \mathrm{A}}^{\mathrm{kSt}}$ auf kantonaler bzw. kommunaler Ebene ${ }^{10}$ variieren zwischen ca. 12\% (Stans) und 35\% (Lausanne). Im Durchschnitt liegen die Werte zwischen $15 \%$ und $25 \%$ (für die Ermittlung dieser Steuersätze wurden jeweils die Grundtarife in der obersten Steuerprogression der jeweiligen Kantone zugrunde gelegt). Der Gesamtsteuersatz der Einkommensteuer im Kanton B ergibt sich aus der Summe von Bundessteuersatz und dem Steuersatz auf kantonaler bzw. kommunaler Ebene.

$$
\begin{aligned}
& \mathrm{s}_{\mathrm{ESt}, \mathrm{B}}=\mathrm{s}_{\mathrm{ESt}}^{\mathrm{BSt}}+\mathrm{s}_{\mathrm{ESt}, \mathrm{B}}^{\mathrm{kkSt}} \\
& \text { mit: } \\
& \mathrm{S}_{\mathrm{ESt}, \mathrm{B}} \quad \text { Gesamtsteuersatz der Einkommensteuer im Kanton B } \\
& \mathrm{S}_{\mathrm{ESt}}^{\mathrm{BSt}} \quad \text { Einkommensteuersatz auf Bundesebene }(11,5 \%) \\
& \mathrm{s}_{\mathrm{ESt}, \mathrm{B}}^{\mathrm{kkSt}} \quad \text { Einkommensteuersatz auf kantonaler/kommunaler Ebene im Kanton B }
\end{aligned}
$$

Die gesamte Steuerbelastung auf Unternehmens- und Anteilseignerebene lässt sich für die drei unterschiedlichen Fallkonstellationen wie folgt zu kombinierten Grenzsteuersätzen zusammenfassen:

9 Vor Umsetzung der Unternehmenssteuerreform II fanden sich bei ca. der Hälfte der Kantone die unterschiedlichsten Erleichterungen, sobald eine Beteiligung mit einer gewissen prozentualen Höhe vorlag. Teilweise war eine Beteiligung i. H. v. 5\% ausreichend, um in den Genuss einer Steuerermässigung zu kommen (z.B. Luzern, Schwyz, Nidwalden, Zug), teilweise wurde eine Beteiligungshöhe von 10\% gefordert (z.B. Glarus, St. Gallen), zum Teil mindestens 20\% (z.B. Obwalden, Schaffhausen). Ebenso variierten die angewandten Methoden sehr stark: Beispielsweise erfolgte in Luzern, Obwalden und Nidwalden eine Besteuerung zum halben Steuersatz, während in Schwyz ein Viertel des Steuersatzes und in Glarus ein Fünftel des Steuersatzes zur Anwendung kam.

10 Die einzelnen Kantone haben im Regelfall keinen direkt anwendbaren Tarif. Einen festen Tarif wenden nur Basel-Landschaft, Tessin und Wallis an. In den anderen Kantonen setzt sich das Steuermass vielmehr aus einem gesetzlich festgelegten Steuersatz und einem periodisch neu definierten Steuerfuss zusammen. Die effektiv geschuldete Kantonssteuer ergibt sich erst durch Multiplikation der sog. einfachen Steuer (gesetzlich festgelegt) mit dem Steuerfuss. Kommunale Steuern werden regelmässig durch einen Zuschlag auf die kantonale Steuer erhoben (kommunaler Steuerfuss) oder sind bereits bei der kantonalen Steuer inbegriffen. Dieser Steuerfuss ist variabel und variiert in den verschiedenen Veranlagungszeiträumen. 
$\mathrm{s}_{\mathrm{EK}}^{\text {natP }}=1-\left(1-\mathrm{s}_{\mathrm{Kap}, \mathrm{A}}\right)\left(1-\mathrm{x}_{\mathrm{B}} \times \mathrm{s}_{\mathrm{ESt}, \mathrm{B}}\right)$

wobei der Parameter $\mathrm{x}_{\mathrm{B}}$ in Abhängigkeit von der Beteiligungsausgestaltung folgende Werte annimmt:

a) Beteiligung $<10 \%$ (Streubesitz, Besteuerung zu 100\%):

$\mathrm{x}_{\mathrm{SB}, \mathrm{B}}=1$

b) Beteiligung $\geq 10 \%$ im Geschäftsvermögen (Besteuerung zu 50\%):

$\mathrm{x}_{\mathrm{GV}, \mathrm{B}}=0,5$

c) Beteiligung $\geq 10 \%$ im Privatvermögen (Besteuerung zu 60\%):

$\mathrm{x}_{\mathrm{PV}, \mathrm{B}}=0,6$

Die Eigenkapitalanteile unterliegen in den Kantonen und Gemeinden der Vermögensteuer zum Verkehrswert, d.h. bei an der Börse gehandelten Wertpapieren gilt der Börsenkurs, nicht börsennotierte Anteile werden mit einem Schätzwert erfasst. Allerdings gilt auch hier, dass ab einer gewissen Beteiligungshöhe Erleichterungen vorgesehen sind. Entsprechend oben getroffener Annahmen wird die Vermögensteuer aufgrund ihrer geringen Bedeutung nicht in die folgenden Berechnungen mit einbezogen.

\subsubsection{Kapitalgesellschaft als Anteilseigner}

\subsection{Beteiligungsgesellschaften}

Ist der Dividendenempfänger eine weitere Kapitalgesellschaft (Eignerkapitalgesellschaft), so kommt diese sowohl auf Bundesebene als auch in den Kantonen in den Genuss einer Gewinnsteuerermässigung durch einen sog. Beteiligungsabzug. Anders als in vielen anderen nationalen Steuerrechtsordnungen ${ }^{11}$ oder im internationalen Kontext (Doppelbesteuerungsabkommen sehen zur Milderung der Doppelbesteuerung bei Schachtelbeteiligungen regelmässig ein Schachtelprivileg in Form der Dividendenfreistellung vor, siehe z.B. Übersicht bei Vogel/Lehner 2003, Art. 23 Rn. 16.) üblich, erfolgt im schweizerischen Steuerrecht keine direkte Freistellung von Beteiligungserträgen, sondern wird eine Erleichterung für Schachteldividenden indirekt über eine Freistellung der Steuer selbst erreicht.

Gem. Art. 69 DBG ermässigt sich die Gewinnsteuer im Verhältnis des Nettoertrages aus dieser Beteiligung zum Reingewinn, wenn eine Kapitalgesellschaft zu mindestens 20\% an einer anderen Gesellschaft beteiligt ist oder ihre Beteiligung einen Verkehrswert von mindestens 2.000.000 CHF ausmacht. ${ }^{12}$ Nach Art. 70 DBG entspricht der Nettoertrag aus Beteiligungen dem Ertrag dieser Beteiligungen abzüglich des darauf entfallenden Finanzierungsaufwandes und eines Beitrages von 5\% zur Deckung des Verwaltungsaufwandes. ${ }^{13}$ Die Vorgehensweise wird verdeutlicht in Beispiel 1.

\section{Beispiel 1: Beteiligungsabzug}

Eine Kapitalgesellschaft erzielt einen Reingewinn von 2.000.000 CHF. Aus einer 20\%igen Beteiligung an einer weiteren Kapitalgesellschaft erhält sie eine Dividende in Höhe von 500.000 CHF. Finanzierungskosten fallen nicht an.

11 Z. B. das Schachtelprivileg im deutschen Steuerrecht ( $\$ 8 \mathrm{~b}$ deutsches $\mathrm{KStG}$ ) oder die sog. „dividend received deduction" im US-amerikanischen Steuerrecht.

12 Durch die Unternehmenssteuerreform II wird dieser Beteiligungsabzug seit dem 1.1.2011 erweitert: Gem. Art. 69 DBG n. F. bzw. Art. 28 StHG n. F. darf nun der Beteiligungsabzug bereits vorgenommen werden, wenn die Kapitalgesellschaft (i) zu mindestens 10\% am Kapital beteiligt ist, (ii) zu mindestens $10 \%$ am Gewinn und den Reserven beteiligt ist oder (iii) Beteiligungsrechte im Verkehrswert von mindestens 1.000.000 CHF hält. Vgl. $\operatorname{SSK}(2009$, S. 2).

13 Vgl. zum Beteiligungsabzug z. B. Kubaile (2006, 98), Weigell (2008). 
Der Nettoertrag aus der Dividende beträgt (abzügl. 5\% fiktive Verwaltungsspesen): 475.000 CHF.

Die Steuer, die die Dividenden empfangende Kapitalgesellschaft normalerweise auf den Reingewinn von 2.000.000 CHF zahlen müsste, reduziert sich durch den Beteiligungsabzug nach Art. 69 DBG um:

$\frac{\text { Nettoertrag aus Beteiligung } * 100 \%}{\text { gesamter Reingewinn }}=\frac{475.000 * 100 \%}{2.000 .000}=23,75 \%$

Damit ergibt sich z.B. folgende Steuerlast auf Bundesebene unter Verwendung des effektiven Bundessteuersatzes von 7,83\% (Der nominale Bundessteuersatz von 8,5\% wird durch die Abzugsfähigkeit von sich selbst vermindert: $\left.\frac{0,085}{1+0,085}=0,0783\right)$ :

\begin{tabular}{lrr} 
Steuer auf den Reingewinn: & $2.000 .000 \mathrm{CHF} * 0,0783=$ & $156.600 \mathrm{CHF}$ \\
. .. Beteiligungsabzug: & $156.600 \mathrm{CHF} * 0,2375=$ & $37.193 \mathrm{CHF}$ \\
\hline geschuldete Steuer & & $119.407 \mathrm{CHF}$
\end{tabular}

Aus ökonomischer Sicht ergibt sich im Gewinnfall14 hierbei das gleiche wirtschaftliche Ergebnis wie bei einer direkten Dividendenfreistellung (siehe Beispiel 2).

\section{Beispiel 2: Dividendenfreistellung}

Würde das schweizerische Steuerrecht für die in Beispiel 1 dargestellte Kapitalgesellschaft unter gleichbleibenden Voraussetzungen eine 95\%ige Dividendenfreistellung (bzw. eine $100 \%$ ige Freistellung unter Annahme von 5\% nicht abzugsfähigen Verwaltungskosten) vorsehen, würde die Ermittlung der Steuerlast zu einem identischen Ergebnis führen:

\begin{tabular}{|c|c|c|}
\hline Reingewinn: & & 2.000.000 CHF \\
\hline ./. Dividendenfreistellung & $0,95 * 500.000 \mathrm{CHF}$ & 475.000 CHF \\
\hline$=$ Steuerbemessungsgrundlage & & 1.525.000 CHF \\
\hline geschuldete Steuer & $0,0783 * 1.525 .000 \mathrm{CHF}$ & 119.407 CHF \\
\hline
\end{tabular}

Unterschiedliche Ergebnisse in Beispiel 1 und Beispiel 2 ergeben sich nur im Verlustfall, da beim Beteiligungsabzug die Steuer reduziert wird - und nicht wie bei einer Dividendenfreistellung die Bemessungsgrundlage. Annahmegemäss wird vorliegend jedoch von einer Gewinnsituation ausgegangen, der Grenzsteuersatz kann daher ebenso mit einer 95\%igen Steuerfreistellung ermittelt werden. Die kantonalen Steuergesetze folgen dem Bundessteuerrecht, d.h. der Beteiligungsabzug erfolgt in gleicher Weise wie auf Bundesebene (Art. 28 StHG).

Leitet die Kapitalgesellschaft die erhaltenen Dividenden an ihre Anteilseigner weiter, hängen die Steuerfolgen wiederum davon ab, ob dieser ein Privatanleger ist oder die Beteiligung im Geschäftsvermögen hält. Hier sei auf die Ausführungen unter 3.1.3.1 verwiesen.

14 Da beim Beteiligungsabzug die Steuer reduziert wird - und nicht wie etwa bei einer Dividendenfreistellung die Bemessungsgrundlage - ergeben sich im Verlustfall unterschiedliche Ergebnisse. 
Der kombinierte Steuersatz stellt sich daher folgendermassen dar:

$\mathrm{s}_{\mathrm{EK}}^{\mathrm{Kap}}=1-\left(1-\mathrm{s}_{\mathrm{Kap}, \mathrm{A}}\right)\left(1-0,05 \mathrm{~s}_{\mathrm{Kap}, \mathrm{B}}\right)\left(1-\mathrm{x}_{\mathrm{B}} \times \mathrm{s}_{\mathrm{ESt}, \mathrm{B}}\right)$

Auch im Kanton B setzt sich der Steuersatz auf Ebene der Kapitalgesellschaft wieder zusammen aus Bundessteuer und kantonaler bzw. kommunaler Steuer:

$\mathrm{s}_{\mathrm{Kap}, \mathrm{B}}=\frac{0,085+\mathrm{s}_{\mathrm{Kap}, \mathrm{B}}^{\mathrm{kkSt}}}{1,085+\mathrm{s}_{\mathrm{Kap}, \mathrm{B}}^{\mathrm{kkSt}}}$

mit:

$\mathrm{S}_{\text {Kap,B }} \quad$ Effektivsteuersatz der Kapitalgesellschaft im Kanton B

$\mathrm{s}_{\text {Kap,B }}^{\mathrm{kkSt}} \quad$ Effektivsteuersatz für Kapitalgesellschaften auf kantonaler/kommunaler Ebene im Kanton B

\subsection{Holdinggesellschaften}

Die kantonale Steuergesetzgebung kennt in Art. 28 Abs. 2 StHG darüber hinaus noch umfassendere Steuerbefreiungen für Holdinggesellschaften: Kapitalgesellschaften, deren statutarischer Zweck zur Hauptsache in der dauernden Verwaltung von Beteiligungen an anderen Unternehmen besteht und die in der Schweiz keine Geschäftstätigkeit ausüben, sind von der Gewinnsteuer gänzlich steuerbefreit, wenn sich ihre Aktiva entweder zu mindestens zwei Dritteln aus Beteiligungen zusammensetzen oder die Gesellschaftserträge zu mindestens zwei Dritteln aus Beteiligungserträgen bestehen. Auf Bundesebene gibt es keine vergleichbare Regelung im DBG.

Kapitalgesellschaften, die diese Voraussetzungen für das Holding-Privileg nicht erfüllen, können jedoch weiterhin den regulären Beteiligungsabzug vornehmen, sofern sie das Erfordernis einer mindestens 20\%igen Beteiligung erfüllen. Damit ist die Bedeutung dieser Holding-Regelung gering, da der Beteiligungsabzug nach obiger Formel bei einer hohen Beteiligung sowieso gegen 100\% tendiert. Dass es dieses Holdingprivileg auf Bundesebene nicht gibt, macht sich damit nur bemerkbar bei Holdinggesellschaften, deren Aktiva/Erträge nicht zu 100\%, sondern nur zu 2/3 aus Beteiligungen bzw. Beteiligungserträgen bestehen.

Dem Holdingprivileg kommt damit lediglich im Fall von Streubesitz höhere Bedeutung zu, da dann die Voraussetzungen des Beteiligungsabzugs nicht erfüllt sind. Vorliegend wird jedoch davon ausgegangen, dass sich die Eignerkapitalgesellschaft zu mindestens 20\% an der Bewertungsgesellschaft beteiligt.

\subsection{Steuerliche Belastung von Fremdkapitalgeber-Einkünften}

Wird der Bewertungskapitalgesellschaft Fremdkapital zur Verfügung gestellt, erfolgt der Rückfluss aus dem Kapital in Form von Zinsen.

Wird das Darlehen von einer natürlichen Person gewährt, unterliegen die Zinsen als Einkommen aus beweglichem Vermögen gem. Art. 20 Abs. 1 DBG und Art. 7 StHG der Besteuerung. Diese werden - anders als die Dividenden - vollumfänglich besteuert. Bezüglich des Einkommensteuertarifs kann auf die obigen Ausführungen (3.1.3.1) verwiesen werden, d.h. es ist auf Bundesebene der Grenzsteuersatz gem. Art. 214 DBG anzuwenden (11,5\%), auf kantonaler bzw. kommunaler Ebene die stark variierenden Effektivsteuersätze.

Die Steuerlast auf die Zinseinkünfte ergibt sich also aus: 
$\mathrm{S}_{\mathrm{FK}}^{\mathrm{natP}, \mathrm{C}}=\mathrm{Z} \times \mathrm{S}_{\mathrm{ESt}, \mathrm{C}}$

mit:

Z Zinsaufwand

$\mathrm{s}_{\mathrm{ESt}, \mathrm{C}} \quad$ Gesamtsteuersatz der Einkommensteuer im Kanton C

Der Grenzsteuersatz der Einkommensteuer im Kanton C ergibt sich dabei wieder aus:

$\mathrm{s}_{\mathrm{ESt}, \mathrm{C}}=\mathrm{s}_{\mathrm{ESt}}^{\mathrm{BSt}}+\mathrm{s}_{\mathrm{ESt}, \mathrm{C}}^{\mathrm{kkSt}}$

Handelt es sich beim Zinsempfänger um eine Kapitalgesellschaft (Gläubigerkapitalgesellschaft), geht das Entgelt regulär in deren ertragsteuerliche Bemessungsgrundlage mit ein und unterliegt gänzlich der Körperschaftsteuer - sowohl auf Bundes- als auch auf kantonaler/kommunaler Ebene. Bezüglich der Zusammensetzung des Tarifs kann hier auf die obigen Ausführungen (3.1.1) verwiesen werden.

Die Steuerlast der Fremdkapital überlassenden Kapitalgesellschaft ermittelt sich aus:

$\mathrm{S}_{\mathrm{FK}}^{\mathrm{Kap}, \mathrm{C}}=\mathrm{Z} \times \mathrm{S}_{\mathrm{Kap}, \mathrm{C}}$

Für den Steuersatz auf Ebene der Kapitalgesellschaft im Kanton C gilt:

$\mathrm{s}_{\mathrm{Kap}, \mathrm{C}}=\frac{0,085+\mathrm{s}_{\mathrm{Kap}, \mathrm{C}}^{\mathrm{kkSt}}}{1,085+\mathrm{s}_{\mathrm{Kap}, \mathrm{C}}^{\mathrm{kkSt}}}$

\section{Ermittlung und Analyse des Tax Shields für die unterschiedlichen Fallkonstellationen}

Der sich aus der Fremdfinanzierung ergebende Steuervorteil kann quantifiziert werden durch Gegenüberstellung des Nachsteuereinkommens von Investoren einer teilweise fremdfinanzierten Unternehmung und dem Nachsteuereinkommen von Investoren einer gänzlich eigenfinanzierten Unternehmung: Die Vergleichsunternehmen unterscheiden sich für die Ermittlung des Tax Shields lediglich durch ihre Finanzierung, operative und investive Tätigkeit bleiben identisch.

\subsection{Ableitung der Tax Shields}

Wie oben erläutert ergibt sich in Abhängigkeit von der Rechtsform der Eigentümer (f) folgendes Eigenkapitaleinkommen einer verschuldeten Unternehmung:

$$
\mathrm{Y}_{\mathrm{Ek}, 1}=(\mathrm{G}-\mathrm{Z})\left(1-\mathrm{s}_{\mathrm{Kap}, \mathrm{A}}\right)\left(1-\mathrm{f} \times \mathrm{s}_{\mathrm{Kap}, \mathrm{B}}\right)\left(1-\mathrm{x}_{\mathrm{B}} \times \mathrm{s}_{\mathrm{ESt}, \mathrm{B}}\right)
$$


mit:

$\mathrm{Y}_{\mathrm{Ek}, 1} \quad$ Eigenkapitaleinkommen aus einer verschuldeten Unternehmung

G Reingewinn vor Zinsen und Steuern

$\mathrm{f}= \begin{cases}0,05 & \text { falls Eigenkapitalgeber eine Kapitalgesellschaft ist } \\ 0 & \text { falls Eigenkapitalgeber eine natürliche Person ist }\end{cases}$

Das Einkommen der Fremdkapitalgeber beträgt in Abhängigkeit von ihrer Rechtsform:

$\mathrm{Y}_{\mathrm{FK}}=\mathrm{Z} \times\left(1-\mathrm{s}_{\mathrm{p}, \mathrm{C}}\right)$

mit:

$\mathrm{s}_{\mathrm{p}, \mathrm{C}}= \begin{cases}\mathrm{s}_{\mathrm{ESt}, \mathrm{C}} & \text { falls Fremdkapitalgeber eine natürliche Person ist } \\ 1-\left(1-\mathrm{s}_{\mathrm{Kap}, \mathrm{C}}\right)\left(1-\mathrm{x}_{\mathrm{C}} \mathrm{s}_{\mathrm{ESt}, \mathrm{C}}\right) & \text { falls Fremdkapitalgeber eine Kapitalgesellschaft ist }\end{cases}$

Das Gesamteinkommen der Investoren (also Eigen- und Fremdkapitalgeber) im Verschuldungsfall stellt sich dar als:

$$
\begin{aligned}
& \mathrm{Y}_{\mathrm{GK}, 1}=\mathrm{Y}_{\mathrm{EK}, 1}+\mathrm{Y}_{\mathrm{FK}} \\
& \Leftrightarrow \quad \mathrm{Y}_{\mathrm{GK}, \mathrm{l}}=(\mathrm{G}-\mathrm{Z})\left(1-\mathrm{s}_{\mathrm{Kap}, \mathrm{A}}\right)\left(1-\mathrm{f} \times \mathrm{s}_{\mathrm{Kap}, \mathrm{B}}\right)\left(1-\mathrm{x}_{\mathrm{B}} \times \mathrm{s}_{\mathrm{ESt}, \mathrm{B}}\right)+\mathrm{Z}\left(1-\mathrm{s}_{\mathrm{p}, \mathrm{C}}\right) \\
& \Leftrightarrow \quad \mathrm{Y}_{\mathrm{GK}, \mathrm{l}}=\mathrm{G}\left(1-\mathrm{s}_{\mathrm{Kap}, \mathrm{A}}\right)\left(1-\mathrm{f} \times \mathrm{s}_{\mathrm{Kap}, \mathrm{B}}\right)\left(1-\mathrm{x}_{\mathrm{B}} \mathrm{s}_{\mathrm{ESt}, \mathrm{B}}\right)+\mathrm{Z}\left[\left(1-\mathrm{s}_{\mathrm{p}, \mathrm{C}}\right)-\left(1-\mathrm{s}_{\mathrm{Kap}, \mathrm{A}}\right)\left(1-\mathrm{f} \times \mathrm{s}_{\mathrm{Kap}, \mathrm{B}}\right)\left(1-\mathrm{x}_{\mathrm{B}} \mathrm{s}_{\mathrm{ESt}, \mathrm{B}}\right)\right]
\end{aligned}
$$

mit:

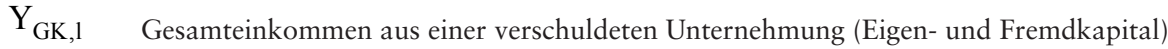

Das Gesamteinkommen des Anteilseigners im Falle einer unverschuldeten Unternehmung ergibt sich aus:

$$
\mathrm{Y}_{\mathrm{Ek}, \mathrm{u}}=\mathrm{G}\left(1-\mathrm{s}_{\mathrm{Kap}, \mathrm{A}}\right)\left(1-\mathrm{f} \times \mathrm{s}_{\mathrm{Kap}, \mathrm{B}}\right)\left(1-\mathrm{x}_{\mathrm{B}} \mathrm{s}_{\mathrm{ESt}, \mathrm{B}}\right)
$$

mit:

$\mathrm{Y}_{\mathrm{Ek}, \mathrm{u}} \quad$ Eigenkapitaleinkommen aus einer unverschuldeten Unternehmung

Damit kann das Tax Shield ermittelt werden:

$$
\begin{aligned}
& \mathrm{TS}=\mathrm{Y}_{\mathrm{GK}, \mathrm{l}}-\mathrm{Y}_{\mathrm{EK}, \mathrm{u}} \\
& \Leftrightarrow \mathrm{TS}=\mathrm{Z}\left[\left(1-\mathrm{s}_{\mathrm{p}, \mathrm{C}}\right)-\left(1-\mathrm{s}_{\mathrm{Kap}, \mathrm{A}}\right)\left(1-\mathrm{f} \times \mathrm{s}_{\mathrm{Kap}, \mathrm{B}}\right)\left(1-\mathrm{x}_{\mathrm{B}} \mathrm{s}_{\mathrm{ESt}, \mathrm{B}}\right)\right] \\
& \Leftrightarrow \mathrm{TS}=\mathrm{Z} \times \mathrm{s}^{*}
\end{aligned}
$$


mit:

TS Tax Shield

$\mathrm{s}^{*} \quad=\left(1-\mathrm{s}_{\mathrm{p}, \mathrm{C}}\right)-\left(1-\mathrm{s}_{\mathrm{Kap}, \mathrm{A}}\right)\left(1-\mathrm{f} \times \mathrm{s}_{\mathrm{Kap}, \mathrm{B}}\right)\left(1-\mathrm{x}_{\mathrm{B}} \mathrm{S}_{\mathrm{ESt}, \mathrm{B}}\right)$

Der Prozentsatz des Tax Shields s* ergibt sich damit - wie auch in der Literatur für ein einfaches Steuersystem dargestellt (vgl. stellvertretend Berk/DeMarzo 2007, 489ff.) - aus der Differenz des Nachsteuerzuflusses auf Ebene des Fremdkapitalgebers (hier repräsentiert durch $\left(1-s_{p, C}\right)$ ) und dem Nachsteuerzufluss auf Ebene des Eigenkapitalgebers (hier repräsentiert durch $\left.\left(1-\mathrm{f} \times \mathrm{s}_{\mathrm{Kap}, \mathrm{B}}\right)\left(1-\mathrm{x}_{\mathrm{B}} \mathrm{s}_{\mathrm{ESt}, \mathrm{B}}\right)\right)$ unter Berücksichtigung der Steuerersparnis auf Gesellschaftsebene $\left(1-\mathrm{s}_{\mathrm{Kap}, \mathrm{A}}\right) .{ }^{15}$ Mit dieser Definition für $\mathrm{s}$ * sind alle Formeln, die im „Lehrbuchfall“ für ein einfaches Steuersystem entwickelt wurden, unmittelbar anwendbar. ${ }^{16}$

Das Tax Shield stellt im Rahmen der Unternehmensbewertung den durch den Steuervorteil der Fremdfinanzierung ausgelösten Wertbeitrag dar (siehe z. B. Brealey et al. 2008, 503ff.). Der Prozentsatz s* gibt diesen Steuervorteil bezogen auf die Zinsaufwendungen an.

\subsection{Analyse des Tax Shields}

Das Tax Shield wirkt in der Unternehmensbewertung gegenüber dem Basisfall der reinen Eigenfinanzierung unmittelbar werterhöhend, wie in Abschnitt 5 formal ausgeführt ist. Wie der sog. Adjusted Present Value (APV)-Ansatz deutlich macht, ist der Barwert der Tax Shields eine eigene Wertkomponente. Da sich das Tax Shield multiplikativ aus dem Zinsaufwand und dem Satz s* ergibt, führt ein höherer Satz für s* bei gegebenem Zinsaufwand unmittelbar zu höheren Unternehmenswerten. Im Folgenden wird die Höhe dieses Satzes auf seine wesentlichen Einflussgrössen näher untersucht.

\subsubsection{Analyse für den Fall der natürlichen Person als Eigenkapitalgeber}

Wird davon ausgegangen, dass der Fremdkapitalgeber eine natürliche Person ist und weiterhin, dass sich die Kapitalgesellschaftsanteile im Geschäftsvermögen der natürlichen Person befinden (also hälftige Besteuerung von Dividenden mit $\mathrm{x}_{\mathrm{GV}, \mathrm{B}}=0,5$ ), ergeben sich unter Annahme eines Einkommensteuersatzes im Kanton C ( $\left.\mathrm{s}_{\mathrm{ESt}, \mathrm{C}}^{\mathrm{kkSt}}\right)$ von $10 \%$ beispielsweise folgende Werte (Tabelle 1) für den Prozentsatz des Tax Shields:

15 Berz/DeMarzo $(2007,491)$ geben den Vorteil der Fremdfinanzierung im Verhältnis zur Eigenfinanzierung in relativer Form an. Mit den hier verwendeten Parametern müsste für eine äquivalente Darstellung die Definition von $s *$ durch den Faktor $\left(1-\mathrm{s}_{\mathrm{p}, \mathrm{C}}\right)$ dividiert werden.

16 Diese vereinfachte Darstellung verdanken wir dem Hinweis eines anonymen Gutachters. Die Definitionen für $\mathrm{f}$ (in Formel 10) und für $\mathrm{s}_{\mathrm{p}, \mathrm{C}}$ (in Formel 11) wurden aufgrund des konkreten Vorschlags in seinem Gutachten eingeführt. Mit den entsprechenden Definitionen für $\mathrm{r}_{\mathrm{FK}}^{*}$ und $\mathrm{s}^{*}$ konnte das Tax Shield hierdurch in der vorliegenden Form als Produkt von Zinsaufwand und Steuersatz dargestellt werden. Die Komplexität der Darstellung wurde damit erheblich reduziert. Wir danken dem Gutachter sehr für den konstruktiven Vorschlag. 


\begin{tabular}{|c|c|c|c|c|c|c|c|c|c|}
\hline \multirow[t]{2}{*}{$s^{*}$} & & \multicolumn{8}{|l|}{$\mathrm{S}_{\mathrm{Kap}, \mathrm{A}}^{\mathrm{kkSt}}$} \\
\hline & & $5 \%$ & $10 \%$ & $15 \%$ & $20 \%$ & $25 \%$ & $30 \%$ & $35 \%$ & $40 \%$ \\
\hline \multirow{5}{*}{$\mathrm{s}_{\mathrm{ESt}, \mathrm{B}}^{\mathrm{kkSt}}$} & $10 \%$ & $-0,13 \%$ & $3,18 \%$ & $6,23 \%$ & $9,04 \%$ & $11,65 \%$ & $14,06 \%$ & $16,30 \%$ & $18,40 \%$ \\
\hline & $15 \%$ & $2,07 \%$ & $5,29 \%$ & $8,26 \%$ & $10,99 \%$ & $13,52 \%$ & $15,86 \%$ & $18,05 \%$ & $20,08 \%$ \\
\hline & $20 \%$ & $4,27 \%$ & $7,40 \%$ & $10,28 \%$ & $12,94 \%$ & $15,39 \%$ & $17,67 \%$ & $19,79 \%$ & $21,77 \%$ \\
\hline & $25 \%$ & $6,47 \%$ & $9,51 \%$ & $12,31 \%$ & $14,88 \%$ & $17,26 \%$ & $19,47 \%$ & $21,53 \%$ & $23,45 \%$ \\
\hline & $30 \%$ & $8,68 \%$ & $11,62 \%$ & $14,33 \%$ & $16,83 \%$ & $19,14 \%$ & $21,28 \%$ & $23,27 \%$ & $25,13 \%$ \\
\hline
\end{tabular}

Tabelle 1: Prozentsätze des TS für den Fall einer natürlichen Person als Eigenkapitalgeber (Anteile im GV) und als Fremdkapitalgeber $\left(\mathrm{x}_{\mathrm{GV}, \mathrm{B}}=0,5 ; \mathrm{s}_{\mathrm{ESt}, \mathrm{C}}^{\mathrm{kkS}}=10 \%\right)$

Befinden sich - unter ansonsten gleichbleibenden Bedingungen - die Anteile hingegen in Streubesitz (d.h. volle Dividendenbesteuerung mit $\mathrm{x}_{\mathrm{SB}, \mathrm{B}}=1$ ), ändert sich die Vorteilhaftigkeit der Fremdfinanzierung zu den Werten in Tabelle 2:

\begin{tabular}{|c|c|c|c|c|c|c|c|c|c|}
\hline \multirow[t]{2}{*}{$\mathrm{s}^{*}$} & & \multicolumn{8}{|l|}{$\mathrm{S}_{\mathrm{Kap}, \mathrm{A}}^{\mathrm{kkSt}}$} \\
\hline & & $5 \%$ & $10 \%$ & $15 \%$ & $20 \%$ & $25 \%$ & $30 \%$ & $35 \%$ & $40 \%$ \\
\hline \multirow{5}{*}{$\mathrm{s}_{\mathrm{ESt}, \mathrm{B}}^{\mathrm{kkSt}}$} & $10 \%$ & $9,34 \%$ & $12,26 \%$ & $14,94 \%$ & $17,41 \%$ & $19,70 \%$ & $21,82 \%$ & $23,80 \%$ & $25,64 \%$ \\
\hline & $15 \%$ & $13,74 \%$ & $16,47 \%$ & $18,99 \%$ & $21,30 \%$ & $23,44 \%$ & $25,43 \%$ & $27,28 \%$ & $29,01 \%$ \\
\hline & $20 \%$ & $18,15 \%$ & $20,69 \%$ & $23,03 \%$ & $25,19 \%$ & $27,19 \%$ & $29,04 \%$ & $30,76 \%$ & $32,37 \%$ \\
\hline & $25 \%$ & $22,55 \%$ & $24,91 \%$ & $27,08 \%$ & $29,08 \%$ & $30,93 \%$ & $32,65 \%$ & $34,25 \%$ & $35,74 \%$ \\
\hline & $30 \%$ & $26,96 \%$ & $29,13 \%$ & $31,13 \%$ & $32,97 \%$ & $34,68 \%$ & $36,26 \%$ & $37,73 \%$ & $39,11 \%$ \\
\hline
\end{tabular}

Tabelle 2: Prozentsätze des TS für den Fall einer natürlichen Person als Eigenkapitalgeber (Anteile in Streubesitz) und als Fremdkapitalgeber $\left(\mathrm{x}_{\mathrm{SB}, \mathrm{B}}=1 ; \mathrm{s}_{\mathrm{ESt}, \mathrm{C}}^{\mathrm{kkSt}}=10 \%\right)$

Zunächst wird deutlich, dass die Erleichterung bei der Dividendenbesteuerung erhebliche Relevanz bei der Entstehung des Tax Shields entfaltet: Der Prozentsatz für die Ermittlung des Tax Shields wird durch die Teilbesteuerung von Dividenden nahezu doppelt so hoch.

Ein geringes Steuerniveau im Kanton A - also auf Ebene der Kapital empfangenden Kapitalgesellschaft - verhindert das Entstehen eines umfangreichen Fremdfinanzierungsvorteils, da sich der Betriebsausgabenabzug der Zinsen nicht so stark auswirken kann.

Besteht jedoch gleichzeitig ein hohes Steuerniveau im Kanton B (Kanton des Anteilseigners) und kennt dieser darüber hinaus keine Steuerbefreiung der Dividenden, so kann unter bestimmten Umständen dennoch ein ebenso hohes Tax Shield erzielt werden. So ist z. B. in Tabelle 2 der Prozentwert des TS bei einer Einkommensteuer im B von 30\% und einer Besteuerung auf Kapitalgesellschaftsebene im A von 5\% mit 26,96\% in etwa gleich hoch, wie in dem Fall, dass im A ein Steuersatz von 40\% und im B ein Steuersatz von 30\% angenommen wird $(25,64 \%)$.

Der Steuervorteil der Fremdfinanzierung ist hier jedoch nicht nur auf die steuerliche Zinsabzugsfähigkeit zurückzuführen, sondern beruht vorwiegend auf dem Einkommensteuergefälle zwischen Kanton B und Kanton C.

Der Einfluss des Einkommensteuerniveaus des Fremdkapitalgebers (Kanton C) wird insbesondere auch deutlich, wenn statt der bisher angenommenen 10\% für die Einkommensteuer im C nunmehr 20\% angenommen werden (Tabelle 3): 


\begin{tabular}{|c|c|c|c|c|c|c|c|c|c|}
\hline \multirow[t]{2}{*}{$s^{*}$} & & \multicolumn{8}{|l|}{$\mathrm{S}_{\mathrm{Kap}, \mathrm{A}}^{\mathrm{kkSt}}$} \\
\hline & & $5 \%$ & $10 \%$ & $15 \%$ & $20 \%$ & $25 \%$ & $30 \%$ & $35 \%$ & $40 \%$ \\
\hline \multirow{5}{*}{$\mathrm{s}_{\mathrm{ESt}, \mathrm{B}}^{\mathrm{kkSt}}$} & $10 \%$ & $-10,13 \%$ & $-6,82 \%$ & $-3,77 \%$ & $-0,96 \%$ & $1,65 \%$ & $4,06 \%$ & $6,30 \%$ & $8,40 \%$ \\
\hline & $15 \%$ & $-7,93 \%$ & $-4,71 \%$ & $-1,74 \%$ & $0,99 \%$ & $3,52 \%$ & $5,86 \%$ & $8,05 \%$ & $10,08 \%$ \\
\hline & $20 \%$ & $-5,73 \%$ & $-2,60 \%$ & $0,28 \%$ & $2,94 \%$ & $5,39 \%$ & $7,67 \%$ & $9,79 \%$ & $11,77 \%$ \\
\hline & $25 \%$ & $-3,53 \%$ & $-0,49 \%$ & $2,31 \%$ & $4,88 \%$ & $7,26 \%$ & $9,47 \%$ & $11,53 \%$ & $13,45 \%$ \\
\hline & $30 \%$ & $-1,32 \%$ & $1,62 \%$ & $4,33 \%$ & $6,83 \%$ & $9,14 \%$ & $11,28 \%$ & $13,27 \%$ & $15,13 \%$ \\
\hline
\end{tabular}

Tabelle 3: Prozentsätze des TS für den Fall einer natürlichen Person als Eigenkapitalgeber (Anteile im GV) und als Fremdkapitalgeber bei höherem ESt-Niveau $\left(\mathrm{x}_{\mathrm{GV}, \mathrm{B}}=0,5 ; \mathrm{s}_{\mathrm{ESt}, \mathrm{C}}^{\mathrm{kkSt}}=20 \%\right)$

Ein höheres Einkommensteuerniveau im Kanton C lässt die Werte für das Tax Shield stark sinken: Der Betriebsausgabenabzug auf Ebene der Kapitalgesellschaft im Kanton A wird durch die hohe Besteuerung der Zinseinkünfte im Kanton C kompensiert. Bei einer durchschnittlichen kantonalen/kommunalen Steuerbelastung im Kanton A von 15\% und einer kantonalen/kommunalen Einkommensteuer von 20\% im B wird die Vorteilhaftigkeit nahezu auf $0 \%$ reduziert (hier $0,28 \%$ ).

Insgesamt fällt auf, dass sich die prozentualen Werte für das Tax Shield in einer hohen Bandbreite zwischen sehr geringen bzw. sogar negativen Werten und sehr hohen Werten bewegen.

Anders stellt sich die Situation dar, wenn Fremdkapital von einer Kapitalgesellschaft überlassen wird: Werden beispielsweise gleiche Parameter angenommen wie in Tabelle 3 und darüber hinaus ein Steuersatz auf Ebene der Kapitalgesellschaft im C von 20\% und eine hälftige Dividendenbesteuerung im C, ergeben sich Prozentwerte in Tabelle 4 für das Tax Shield:

\begin{tabular}{|ll|r|r|r|r|r|r|r|r|}
\hline \multirow{2}{*}{$\mathrm{s} *$} & \multicolumn{2}{|c|}{$\mathrm{S}$ KkSt } \\
& & $5 \%$ & $10 \%$ & $15 \%$ & $20 \%$ & $25 \%$ & $30 \%$ & $35 \%$ & $40 \%$ \\
\hline \multirow{3}{*}{$\mathrm{skSt}$} & $10 \%$ & $-9,18 \%$ & $-5,86 \%$ & $-2,81 \%$ & $0,00 \%$ & $2,60 \%$ & $5,01 \%$ & $7,26 \%$ & $9,35 \%$ \\
\cline { 2 - 10 } & $15 \%$ & $-6,98 \%$ & $-3,75 \%$ & $-0,79 \%$ & $1,95 \%$ & $4,47 \%$ & $6,82 \%$ & $9,00 \%$ & $11,04 \%$ \\
\cline { 2 - 10 } & $20 \%$ & $-4,77 \%$ & $-1,64 \%$ & $1,24 \%$ & $3,89 \%$ & $6,35 \%$ & $8,62 \%$ & $10,74 \%$ & $12,72 \%$ \\
\cline { 2 - 10 } & $25 \%$ & $-2,57 \%$ & $0,47 \%$ & $3,26 \%$ & $5,84 \%$ & $8,22 \%$ & $10,43 \%$ & $12,49 \%$ & $14,40 \%$ \\
\cline { 2 - 10 } & $30 \%$ & $-0,37 \%$ & $2,58 \%$ & $5,29 \%$ & $7,78 \%$ & $10,09 \%$ & $12,24 \%$ & $14,23 \%$ & $16,09 \%$ \\
\hline
\end{tabular}

Tabelle 4: Prozentsätze des TS für den Fall einer natürlichen Person als Eigenkapitalgeber (Anteile im GV) und einer Kapitalgesellschaft als Fremdkapitalgeber bei hälftiger Dividendenbesteuerung im C $\left(\mathrm{x}_{\mathrm{GV}, \mathrm{B}}=0,5 ; \mathrm{s}_{\mathrm{ESSt}, \mathrm{C}}^{\mathrm{kSt}}=20 \% ; \mathrm{s}_{\mathrm{Kap}, \mathrm{C}}=20 \% ; \mathrm{x}_{\mathrm{C}}=0,5\right)$

Nimmt man hingegen eine volle Dividendenbesteuerung im Kanton $\mathrm{C}$ an, ändern sich die Prozentsätze zu den in Tabelle 5 aufgeführten Werten.

\begin{tabular}{|ll|r|r|r|r|r|r|r|r|}
\hline \multirow{2}{*}{$\mathrm{s}^{*}$} & \multicolumn{2}{|c|}{$\mathrm{S} \mathrm{k}$ Kap, $\mathrm{A}$} \\
& & $5 \%$ & $10 \%$ & $15 \%$ & $20 \%$ & $25 \%$ & $30 \%$ & $35 \%$ & $40 \%$ \\
\hline \multirow{3}{*}{$\mathrm{s} \mathrm{kkSt}$} & $10 \%$ & $-17,54 \%$ & $-14,23 \%$ & $-11,18 \%$ & $-8,37 \%$ & $-5,76 \%$ & $-3,35 \%$ & $-1,11 \%$ & $0,99 \%$ \\
\cline { 2 - 10 } & $15 \%$ & $-15,34 \%$ & $-12,12 \%$ & $-9,15 \%$ & $-6,42 \%$ & $-3,89 \%$ & $-1,55 \%$ & $0,64 \%$ & $2,67 \%$ \\
\cline { 2 - 10 } & $20 \%$ & $-13,14 \%$ & $-10,01 \%$ & $-7,13 \%$ & $-4,47 \%$ & $-2,02 \%$ & $0,26 \%$ & $2,38 \%$ & $4,36 \%$ \\
\cline { 2 - 10 } & $25 \%$ & $-10,94 \%$ & $-7,90 \%$ & $-5,10 \%$ & $-2,53 \%$ & $-0,15 \%$ & $2,06 \%$ & $4,12 \%$ & $6,04 \%$ \\
\cline { 2 - 10 } & $30 \%$ & $-8,73 \%$ & $-5,79 \%$ & $-3,08 \%$ & $-0,58 \%$ & $1,73 \%$ & $3,87 \%$ & $5,86 \%$ & $7,72 \%$ \\
\hline
\end{tabular}

Tabelle 5: Prozentsätze des TS für den Fall einer natürlichen Person als Eigenkapitalgeber (Anteile im GV) und einer Kapitalgesellschaft als Fremdkapitalgeber bei voller Dividendenbesteuerung im C $\left(\mathrm{x}_{\mathrm{GV}, \mathrm{B}}=0,5 ; \mathrm{s}_{\mathrm{ESt}, \mathrm{C}}^{\mathrm{kkSt}}=20 \% ; \mathrm{s}_{\mathrm{Kap}, \mathrm{C}}=20 \%, \mathrm{x}_{\mathrm{C}}=1\right)$ 
Es wird deutlich, dass durch Zwischenschaltung einer Kapitalgesellschaft beim Fremdkapitalgeber, d.h. wenn das Fremdkapital nicht unmittelbar durch eine natürliche Person, sondern von einer Kapitalgesellschaft überlassen wird, der Steuervorteil der Fremdfinanzierung abnimmt.

Auch hier spielt eine massgebende Rolle, zu welcher Steuerlast die von der Kapitalgesellschaft im Kanton C vereinnahmten Zinsen bei Weiterleitung an die Anteilseigner in Form einer Ausschüttung führen. Der Ausgestaltung des Dividendenbesteuerungssystems im Ansässigkeitskanton der Fremdkapital überlassenden Kapitalgesellschaft kommt damit ebenfalls massgebende Bedeutung zu. Analog zu den Werten in Tabelle 3 gilt auch hier: Je höher die Besteuerung der Fremdkapitalzinsen im Kanton C ist, je niedriger das Steuerniveau im Kanton A ist und je niedriger das Steuerniveau im Kanton B ist, desto geringer fallen die Werte für das Tax Shield aus.

\subsubsection{Analyse für den Fall einer Kapitalgesellschaft als Eigenkapitalgeber}

Wird das Fremdkapital von natürlichen Personen, das Eigenkapital hingegen von einer Kapitalgesellschaft gehalten, ergeben sich unter Annahme der gleichen Parameter wie in Tabelle 1 und einer kantonalen/kommunalen Steuerbelastung auf Ebene der Kapitalgesellschaft im Kanton B von 20\% die in Tabelle 6 ermittelten Tax Shield-Werte:

\begin{tabular}{|ll|r|r|r|r|r|r|r|r|}
\hline \multirow{2}{*}{$\mathrm{s}^{*}$} & \multicolumn{2}{|c|}{$\mathrm{S}_{\mathrm{Kap}, \mathrm{A}}^{\mathrm{kkSt}}$} \\
& & $5 \%$ & $10 \%$ & $15 \%$ & $20 \%$ & $25 \%$ & $30 \%$ & $35 \%$ & $40 \%$ \\
\hline \multirow{3}{*}{$\mathrm{skSt}$} & $10 \%$ & $0,74 \%$ & $4,02 \%$ & $7,03 \%$ & $9,81 \%$ & $12,39 \%$ & $14,77 \%$ & $16,99 \%$ & $19,07 \%$ \\
\cline { 2 - 10 } & $15 \%$ & $2,92 \%$ & $6,11 \%$ & $9,04 \%$ & $11,74 \%$ & $14,24 \%$ & $16,56 \%$ & $18,72 \%$ & $20,73 \%$ \\
\cline { 2 - 10 } & $20 \%$ & $5,09 \%$ & $8,19 \%$ & $11,04 \%$ & $13,66 \%$ & $16,09 \%$ & $18,34 \%$ & $20,44 \%$ & $22,40 \%$ \\
\cline { 2 - 10 } & $25 \%$ & $7,27 \%$ & $10,28 \%$ & $13,04 \%$ & $15,59 \%$ & $17,94 \%$ & $20,13 \%$ & $22,16 \%$ & $24,06 \%$ \\
\cline { 2 - 10 } & $30 \%$ & $9,45 \%$ & $12,36 \%$ & $15,04 \%$ & $17,51 \%$ & $19,80 \%$ & $21,91 \%$ & $23,89 \%$ & $25,72 \%$ \\
\hline
\end{tabular}

Tabelle 6: Prozentsätze des TS für den Fall einer Kapitalgesellschaft als Eigenkapitalgeber und einer natürlichen Person als Fremdkapitalgeber (Anteile im GV) $\left(\mathrm{x}_{\mathrm{GV}, \mathrm{B}}=0,5 ; \mathrm{s}_{\mathrm{Kap}, \mathrm{B}}=20 \%, \mathrm{~s}_{\mathrm{ESt}, \mathrm{C}}^{\mathrm{kkSt}}=10 \%\right.$ )

Die Prozentsätze für den Steuervorteil aus der Fremdfinanzierung weichen nur marginal von den Werten in Tabelle 1 ab. Aufgrund des Beteiligungsabzugs auf Ebene der zwischengeschalteten Kapitalgesellschaft ist das Tax Shield im Fall einer direkten oder indirekten Beteiligung nahezu gleich hoch.

Handelt es sich sowohl bei dem Eigenkapitalgeber als auch bei dem Fremdkapitalgeber um eine Kapitalgesellschaft, ist für die Entstehung eines Steuervorteils das Steuerniveau in allen drei Kantonen A, B und C massgebend.

Bei einem Steuersatz auf Ebene der Kapitalgesellschaft im B von 30\% und im C von $20 \%$ ergeben sich zum Beispiel die in Tabelle 7 aufgeführten Werte:

\begin{tabular}{|ll|r|r|r|r|r|r|r|r|}
\hline \multirow{2}{*}{$\mathrm{s}^{*}$} & \multicolumn{2}{|c|}{$\mathrm{S}$ KkSt } \\
& & $5 \%$ & $10 \%$ & $15 \%$ & $20 \%$ & $25 \%$ & $30 \%$ & $35 \%$ & $40 \%$ \\
\hline \multirow{3}{*}{$\mathrm{skSt}$} & $10 \%$ & $-8,09 \%$ & $-4,81 \%$ & $-1,81 \%$ & $0,97 \%$ & $3,53 \%$ & $5,91 \%$ & $8,12 \%$ & $10,19 \%$ \\
\cline { 2 - 10 } & $15 \%$ & $-5,91 \%$ & $-2,73 \%$ & $0,19 \%$ & $2,88 \%$ & $5,38 \%$ & $7,69 \%$ & $9,84 \%$ & $11,85 \%$ \\
\cline { 2 - 10 } & $20 \%$ & $-3,74 \%$ & $-0,65 \%$ & $2,18 \%$ & $4,80 \%$ & $7,22 \%$ & $9,47 \%$ & $11,56 \%$ & $13,51 \%$ \\
\cline { 2 - 10 } & $25 \%$ & $-1,57 \%$ & $1,43 \%$ & $4,18 \%$ & $6,72 \%$ & $9,07 \%$ & $11,25 \%$ & $13,28 \%$ & $15,17 \%$ \\
\cline { 2 - 10 } & $30 \%$ & $0,60 \%$ & $3,51 \%$ & $6,18 \%$ & $8,64 \%$ & $10,92 \%$ & $13,03 \%$ & $15,00 \%$ & $16,83 \%$ \\
\hline
\end{tabular}

Tabelle 7: Prozentsätze des TS für den Fall einer Kapitalgesellschaft als Eigenkapitalgeber und als Fremdkapitalgeber bei höherem KSt-Niveau im B $\left(\mathrm{x}_{\mathrm{GV}, \mathrm{B}}=0,5 ; \mathrm{s}_{\mathrm{Kap}, \mathrm{B}}^{\mathrm{kkSt}}=30 \%, \mathrm{~s}_{\mathrm{KapC}}^{\mathrm{kkSt}}=20 \% ; \mathrm{s}_{\mathrm{ESt}, \mathrm{C}}^{\mathrm{kkSt}}=10 \%, \mathrm{x}_{\mathrm{C}}=1\right)$ 
Die Werte für das Tax Shield sind wesentlich niedriger als im Fall der Fremdkapitalüberlassung durch eine natürliche Person. Während es im Fall der Eigenfinanzierung also nahezu unerheblich ist, ob das Eigenkapital von natürlichen Personen oder einer zwischengeschalteten Kapitalgesellschaft überlassen wird, spielt die Rechtsform im Fall der Fremdfinanzierung eine erhebliche Rolle: Ein Steuervorteil entsteht vor allem dann, wenn es sich bei dem Kapitalgeber um eine natürliche Person handelt. Stammt das Fremdkapital von einer Kapitalgesellschaft, stellt sich ein positiver Wert für den Steuervorteil der Fremdfinanzierung erst dann ein, wenn im Kanton der Kapital empfangenden Kapitalgesellschaft ein ausreichend hohes Körperschaftsteuerniveau herrscht.

\section{Ableitung der Bewertungsformeln für den APV- und den WACC-Ansatz}

Mit den bislang ermittelten Tax Shields lässt sich nunmehr auch die Discounted CashflowMethodik unter Berücksichtigung eines schweizerischen Steuerumfeldes darstellen.

\subsection{Berücksichtigung im APV-Ansatz}

Nach dem Adjusted-Present-Value (APV)-Ansatz werden die Tax Shields aufgrund der ihr eigenen Risikostruktur gesondert neben den finanzierungsunabhängig ermittelten Zahlungsüberschüssen bewertet. Es erfolgt eine gesonderte Bewertung des Fremdfinanzierungsvorteils und des Unternehmenswertes bei reiner Eigenfinanzierung. Das Einkommen der Investoren einer verschuldeten Unternehmung besteht mithin aus zwei Komponenten: Erstens dem Einkommen, das auch aus einem unverschuldeten Unternehmen erzielbar wäre und zweitens dem Steuervorteil (TS). In der DCF-Methodik wird das erzielbare Einkommen des unverschuldeten Unternehmens über den Brutto-Free Cashflow (BFCF) abgebildet (für eine exakte Definition der BFCF anhand der Kapitalflussrechnung siehe Schultze/Meyer 2005, 289): $\mathrm{BFCF}_{\mathrm{t}}=\mathrm{OCF}_{\mathrm{t}}+\mathrm{ICF}_{\mathrm{t}}-\mathrm{T}_{\mathrm{t}}^{\mathrm{u}}$. Da sein Risiko allein von operativen Risiken bestimmt wird, ist er mit den Kapitalkosten des unverschuldeten Unternehmens zu diskontieren. Der Steuervorteil hingegen hängt von der Höhe der Verschuldung ab, ist im Bewertungszeitpunkt bekannt und ebenso riskant wie das Fremdkapital. Er ist daher für die erste Periode mit den Fremdkapitalkosten zu diskontieren. Für die Folgeperioden ist die Höhe der Steuervorteile von der Finanzierungspolitik abhängig, welche entweder unternehmenswertabhängig festgelegt werden kann (sog. L-Modell) oder im Bewertungszeitpunkt determiniert ist (sog. F-Modell). Im ersten Fall unterliegt die Höhe der Verschuldung und das TS denselben Schwankungen wie der Unternehmenswert und ist daher ebenso riskant (Miles/Ezzel 1980, 724), im zweiten Fall ist er nur von der Höhe der Verschuldung abhängig und mithin gleichermassen riskant wie das Fremdkapital (Wallmeier 1999, 1474).

Insgesamt ergeben sich unter der vereinfachenden Annahme eines ewigen Planungshorizontes folgende Bewertungsformeln für den APV-Ansatz:

L-Modell: $\mathrm{Gk}_{0}^{\ell}=\sum_{\mathrm{t}=1}^{\infty} \frac{\mathrm{OCF}_{\mathrm{t}}+\mathrm{ICF}_{\mathrm{t}}-\mathrm{T}_{\mathrm{t}}^{\mathrm{u}}}{\left(1+\mathrm{r}_{\mathrm{Ek}}^{\mathrm{u}^{*}}\right)^{\mathrm{t}}}+\sum_{\mathrm{t}=1}^{\infty} \frac{\mathrm{TS}_{\mathrm{t}}}{\left(1+\mathrm{r}_{\mathrm{Fk}}^{*}\right)\left(1+\mathrm{r}_{\mathrm{Ek}}^{\mathrm{u}^{*}}\right)^{\mathrm{t}-1}}$

F-Modell: $\mathrm{Gk}_{0}^{\ell}=\sum_{\mathrm{t}=1}^{\infty} \frac{\mathrm{OCF}_{\mathrm{t}}+\mathrm{ICF}_{\mathrm{t}}-\mathrm{T}_{\mathrm{t}}^{\mathrm{u}}}{\left(1+\mathrm{r}_{\mathrm{Ek}}^{\mathrm{u}}\right)^{\mathrm{t}}}+\sum_{\mathrm{t}=1}^{\infty} \frac{\mathrm{TS}_{\mathrm{t}}}{\left(1+\mathrm{r}_{\mathrm{Fk}}^{*}\right)^{\mathrm{t}}}$ 
wobei

$\mathrm{r}_{\mathrm{Ek}}^{\mathrm{u}} \quad$ Eigenkapitalkosten vor pers. Steuern eines unverschuldeten Unternehmens

$r_{E k}^{u^{*}} \quad$ Eigenkapitalkosten nach pers. Steuern eines unverschuldeten Unternehmens

$\mathrm{r}_{\mathrm{Ek}}^{\ell^{*}} \quad$ Eigenkapitalkosten nach pers. Steuern eines verschuldeten Unternehmens

$\mathrm{r}_{\mathrm{Fk}}^{*} \quad$ Fremdkapitalkosten nach pers. Steuern

$\mathbf{r}_{\text {wacc }}^{\ell^{*}} \quad$ Gewichtete Kapitalkosten nach Steuern eines verschuldeten Unternehmens

$\ell \quad$ verschuldet (Index)

u unverschuldet (Index)

Gk Marktwert des Gesamtkapitals

Fk Marktwert des Fremdkapitals

Ek Marktwert des Eigenkapitals

OCF Operativer Cashflow

ICF Investitions-Cashflow

$\mathrm{T}^{\mathrm{u}} \quad$ Gesamte Steuerbelastung der Eigentümer eines unverschuldeten Unternehmens

Basierend auf diesen allgemeinen Bewertungsformeln lassen sich die Bedingungen für die unterschiedlichen Konstellationen in der Schweiz berücksichtigen, indem man jeweils die Steuerbelastungen $\left(\mathrm{T}^{\mathrm{u}}\right)$ und Tax Shields (TS) für die konkreten Rahmenbedingungen im schweizerischen Steuerrecht spezifiziert.

Die Steuerbelastung des unverschuldeten Unternehmens $\mathrm{T}^{\mathrm{u}}$ beträgt für den Fall einer natürlichen Person als Anteilseigner grundsätzlich (Anwendung des Steuersatzes aus Formel (3) auf den Gewinn vor Steuern):

$$
\mathrm{T}^{\mathrm{u}}=\mathrm{G}^{\times} \mathrm{s}_{\mathrm{EK}} \Leftrightarrow \mathrm{T}^{\mathrm{u}}=\mathrm{G} \times\left[1-\left(1-\mathrm{s}_{\mathrm{Kap}, \mathrm{A}}\right)\left(1-\mathrm{f} \times \mathrm{s}_{\mathrm{Kap}, \mathrm{B}}\right)\left(1-\mathrm{x}_{\mathrm{B}} \times \mathrm{s}_{\mathrm{ESt}, \mathrm{B}}\right)\right]
$$

Die Tax Shields ergeben sich aus:

$$
\mathrm{TS}=\mathrm{Z} \times \mathrm{S}^{*}
$$

Es wird deutlich, dass ein höherer Wert von $\mathrm{s}^{*}$ sich unmittelbar steigernd auf den Unternehmenswert auswirkt. Dabei ergibt sich s* - gemäss obigen Ausführungen (Abschnitt 3) - je nach Fallkonstellation wie in Abbildung 4 aufgeführt:

\begin{tabular}{|l|l|}
\hline Natürliche Person als EK-Geber: \\
\hline $\begin{array}{l}\text { FK-Geber: natürliche } \\
\text { Pers. }\end{array}$ & $\mathrm{s}^{*}=\left[\left(1-\mathrm{s}_{\mathrm{ESt}, \mathrm{C}}\right)-\left(1-\mathrm{s}_{\mathrm{Kap}, \mathrm{A}}\right)\left(1-\mathrm{x}_{\mathrm{B}} \mathrm{s}_{\mathrm{ESt}, \mathrm{B}}\right)\right]$ \\
\hline $\begin{array}{l}\text { FK-Geber: Kapitalge- } \\
\text { sellschaft }\end{array}$ & $\mathrm{s}^{*}=\left[\left(1-\mathrm{s}_{\mathrm{Kap}, \mathrm{C}}\right)\left(1-\mathrm{x}_{\mathrm{C}} \mathrm{s}_{\mathrm{ESt}, \mathrm{C}}\right)-\left(1-\mathrm{s}_{\mathrm{Kap}, \mathrm{A}}\right)\left(1-\mathrm{x}_{\mathrm{B}} \mathrm{s}_{\mathrm{ESt}, \mathrm{B}}\right)\right]$ \\
\hline Kapitalgesellschaft als EK-Geber \\
\hline $\begin{array}{l}\text { FK-Geber: natürliche } \\
\text { Pers. }\end{array}$ & $\mathrm{s}^{*}=\left[\left(1-\mathrm{s}_{\mathrm{ESt}, \mathrm{C}}\right)-\left(1-\mathrm{s}_{\mathrm{Kap}, \mathrm{A}}\right)\left(1-0,05 \mathrm{~s}_{\mathrm{Kap}, \mathrm{B}}\right)\left(1-\mathrm{x}_{\mathrm{B}} \mathrm{s}_{\mathrm{ESt}, \mathrm{B}}\right)\right]$ \\
\hline $\begin{array}{l}\text { FK-Geber: Kapitalge- } \\
\text { sellschaft }\end{array}$ & $\mathrm{s}^{*}=\left[\left(1-\mathrm{s}_{\mathrm{Kap}, \mathrm{C}}\right)\left(1-\mathrm{x}_{\mathrm{C}} \mathrm{S}_{\mathrm{ESt}, \mathrm{C}}\right)-\left(1-\mathrm{s}_{\mathrm{Kap}, \mathrm{A}}\right)\left(1-0,05 \mathrm{~s}_{\mathrm{Kap}, \mathrm{B}}\right)\left(1-\mathrm{x}_{\mathrm{B}} \mathrm{s}_{\mathrm{ESt}, \mathrm{B}}\right)\right]$ \\
\hline
\end{tabular}

Abbildung 4: Überblick über die ermittelten Tax Shield Formeln 


\subsection{Berücksichtigung im WACC-Ansatz}

Auch der Weighted Average Cost of Capital (WACC)-Ansatz lässt sich mit den obigen Ergebnissen leicht herleiten. Der WACC-Ansatz, bei dem die finanzierungsunabhängig ermittelten Brutto-Free Cashflows (BFCF) mit den WACC zu diskontieren sind, lautet allgemein und steuersystemunabhängig (Schultze 2004, 775):

$$
\mathrm{Gk}_{0}^{\ell}=\sum_{\mathrm{t}=1}^{\infty} \frac{\mathrm{BFCF}_{\mathrm{t}}}{\left(1+\mathrm{r}_{\mathrm{WACC}}^{\ell^{*}}\right)^{\mathrm{t}}}
$$

wobei: $\mathrm{r}_{\text {wacc }}^{\ell^{*}}=\mathrm{r}_{\mathrm{Ek}}^{\ell^{*}} \frac{\mathrm{Ek}}{\mathrm{Gk}}+\mathrm{r}_{\mathrm{Fk}}^{*}\left(1-\frac{\mathrm{TS}}{\mathrm{r}_{\mathrm{Fk}}^{*} \mathrm{Fk}}\right) \frac{\mathrm{Fk}}{\mathrm{Gk}}$

Mit $\mathrm{TS}=\mathrm{Z} \times \mathrm{s}^{*}=\mathrm{r}_{\mathrm{Fk}} \mathrm{Fk} \times \mathrm{s}^{*}$ und $\mathrm{r}_{\mathrm{Fk}}^{*}=\mathrm{r}_{\mathrm{Fk}}\left(1-\mathrm{s}_{\mathrm{p}, \mathrm{C}}\right)$ gilt für die WACC im schweizerischen Steuersystem:

$$
\mathrm{r}_{\mathrm{wacc}}^{\ell^{*}}=\mathrm{r}_{\mathrm{Ek}}^{\ell^{*}} \frac{\mathrm{Ek}}{\mathrm{Gk}}+\mathrm{r}_{\mathrm{Fk}}^{*}\left(1-\frac{\mathrm{r}_{\mathrm{Fk}} \mathrm{Fk} \times \mathrm{s}^{*}}{\mathrm{r}_{\mathrm{Fk}}\left(1-\mathrm{s}_{\mathrm{p}, \mathrm{C}}\right) \mathrm{Fk}}\right) \frac{\mathrm{Fk}}{\mathrm{Gk}}=\mathrm{r}_{\mathrm{Ek}}^{\ell^{*}} \frac{\mathrm{Ek}}{\mathrm{Gk}}+\mathrm{r}_{\mathrm{Fk}}^{*}\left(1-\frac{\mathrm{s}^{*}}{\left(1-\mathrm{s}_{\mathrm{p}, \mathrm{C}}\right)}\right) \frac{\mathrm{Fk}}{\mathrm{Gk}}
$$

Bzw., wenn man bei der Ermittlung der WACC von Vorsteuerrenditen ausgeht:

$$
\mathrm{r}_{\mathrm{wacc}}^{\ell^{*}}=\mathrm{r}_{\mathrm{Ek}}^{\varepsilon^{*}} \frac{\mathrm{Ek}}{\mathrm{Gk}}+\mathrm{r}_{\mathrm{Fk}}\left(1-\mathrm{s}_{\mathrm{p}, \mathrm{C}}\right)\left(1-\frac{\mathrm{s}^{*}}{\left(1-\mathrm{s}_{\mathrm{p}, \mathrm{C}}\right)}\right) \frac{\mathrm{Fk}}{\mathrm{Gk}}=\mathrm{r}_{\mathrm{Ek}}^{\varepsilon^{*}} \frac{\mathrm{Ek}}{\mathrm{Gk}}+\mathrm{r}_{\mathrm{Fk}}\left(1-\mathrm{s}_{\mathrm{p}, \mathrm{C}}-\mathrm{s}^{*}\right) \frac{\mathrm{Fk}}{\mathrm{Gk}}
$$

Die WACC ergeben sich somit je nach Fallkonstellation durch die in Abbildung 4 angegebenen $\mathrm{s}^{*}$. Hieraus wird deutlich: Ein höherer Wert für $\mathrm{s} *$ wirkt sich somit unmittelbar werterhöhend aus. Durch die in Abschnitt 4.2. vorgestellte Analyse wird deutlich, welche Einflussfaktoren dies begünstigen. Hierbei sind erhebliche Unterschiede im Tax Shield zu verzeichnen. In einigen Konstellationen verschwindet der Steuervorteil gänzlich oder wandelt sich sogar zum Nachteil. Die in der Praxis häufig zu beobachtende pauschale Anwendung eines Durchschnittssteuersatzes ist daher höchst bedenklich und eine der Fallkonstellation angemessene Berücksichtigung ist angezeigt.

Um die Auswirkung auf den Unternehmenswert zu verdeutlichen, wählen wir aus der obigen Analyse exemplarisch zwei Fallkonstellationen aus und setzen sie in die ermittelten Bewertungsformeln ein. Vergleicht man den Maximalwert aus Tabelle 2 von 39,11\% mit dem Minimalwert aus Tabelle 3 von -10,13\%, so ergeben sich (siehe Beispiel 3) für den ersten Fall WACC in Höhe von 9,4\%, im zweiten Fall von 7,7\%. Bei einer ewigen Rente macht dieser Unterschied von 1,7\% einen Unterschied im Unternehmenswert zum Faktor von 12,99 - 10,64 = 2,35 auf den Free Cashflow aus. Bei einem BFCF von 1.000.000 CHF bewirkt dies also einen Wertunterschied von 2.350.000 CHF. Es wird deutlich, dass die Berücksichtigung von Steuern - in Abhängigkeit von Standortwahl von Kapitalgeber und Kapitalnehmer - erhebliche Relevanz für die Bewertung hat. 
Beispiel 3: WACC

Folgende Werte seien gegeben:

$\mathrm{r}_{\mathrm{Ek}}^{\ell *}=13 \%$

$\mathrm{r}_{\mathrm{Fk}}=8 \%$

$\mathrm{Fk} / \mathrm{Gk}=55 \%$

$\mathrm{Ek} / \mathrm{Gk}=45 \%$

Für s* $=10,13 \%$ der Tabelle 3 links oben ergibt sich (bei Übernahme der entsprechenden Parameter):

$\mathrm{r}_{\text {wacc }}^{\ell^{*}}=0,13 \times 0,45+0,08(1-0,285+0,1013) \times 0,55=0,0944$

mit:

$\mathrm{s}_{\mathrm{p}, \mathrm{C}}=\mathrm{s}_{\mathrm{ESt}}^{\mathrm{BSt}}+\mathrm{s}_{\mathrm{ESS}, \mathrm{C}}^{\mathrm{kKSt}}=0,085+0,2=0,285$

Für s* $=39,11 \%$ der Tabelle 2 unten rechts erhält man

$\mathrm{r}_{\text {wacc }}^{\ell *}=0,13 \times 0,45+0,08(1-0,185-0,3911) \times 0,55=0,077$

mit:

$\mathrm{s}_{\mathrm{p}, \mathrm{C}}=\mathrm{s}_{\mathrm{ESt}}^{\mathrm{BSt}}+\mathrm{s}_{\mathrm{ESt}, \mathrm{C}}^{\mathrm{kSt}}=0,085+0,1=0,185$

Der WACC-Ansatz ebenso wie der Equity-Ansatz sind nur bei Kenntnis der auf die geplante Kapitalstruktur angepassten Eigenkapitalkosten eines verschuldeten Unternehmens $\left(\mathrm{r}_{\mathrm{Ek}}^{\ell^{*}}\right)$ anwendbar. Der Zusammenhang zwischen den Kapitalkosten eines unverschuldeten und eines verschuldeten Unternehmens lautet unter der Annahme der ertragswertabhängigen Finanzierung allgemein: ${ }^{17}$

$$
\mathrm{r}_{\mathrm{Ek}}^{\ell *}=\mathrm{r}_{\mathrm{Ek}}^{\ell}\left(1-\mathrm{s}_{\mathrm{E}}\right)=\mathrm{r}_{\mathrm{Ek}}^{\mathrm{u}}\left(1-\mathrm{s}_{\mathrm{E}}\right)+\left[\mathrm{r}_{\mathrm{Ek}}^{\mathrm{u}}\left(1-\mathrm{s}_{\mathrm{E}}\right)-\mathrm{r}_{\mathrm{Fk}}\left(1-\mathrm{s}_{\mathrm{Fk}}\right)\right]\left(1-\frac{\mathrm{TS} / \mathrm{Fk}}{1+\mathrm{r}_{\mathrm{Fk}}\left(1-\mathrm{s}_{\mathrm{Fk}}\right)}\right) \frac{\mathrm{Fk}}{\mathrm{Ek}}
$$

mit:

$\mathrm{s}_{\mathrm{E}} \quad$ pers. Steuersatz für Eigenkapital-Einkommen

$\mathrm{s}_{\mathrm{Fk}} \quad$ pers. Steuersatz für Fremdkapital-Einkommen

Diese ist für die betreffende Fallkonstellation adäquat anzupassen. Mit TS = $\mathrm{Z} \times \mathrm{s}^{*}=\mathrm{r}_{\mathrm{Fk}} \mathrm{Fk} \times \mathrm{s}^{*}$ und $\mathrm{r}_{\mathrm{Fk}}^{*}=\mathrm{r}_{\mathrm{Fk}}\left(1-\mathrm{s}_{\mathrm{p}, \mathrm{C}}\right)$ gilt im schweizerischen Steuersystem:

$\mathrm{r}_{\mathrm{Ek}}^{\ell *}=\mathrm{r}_{\mathrm{Ek}}^{\mathrm{u}^{*}}+\left[\mathrm{r}_{\mathrm{Ek}}^{\mathrm{u}^{*}}-\mathrm{r}_{\mathrm{Fk}}\right]\left(1-\frac{\mathrm{r}_{\mathrm{Fk}} \times \mathrm{s}^{*}}{1+\mathrm{r}_{\mathrm{Fk}}^{*}}\right) \frac{\mathrm{Fk}}{\mathrm{Ek}}$

Für den alternativen Fall der autonomen Finanzierung existiert eine äquivalente Darstellung. Sie lautet (Schultze 2004, 789):

$\mathrm{r}_{\mathrm{Ek}}^{\ell *}=\mathrm{r}_{\mathrm{Ek}}^{\ell}\left(1-\mathrm{s}_{\mathrm{E}}\right)=\mathrm{r}_{\mathrm{Ek}}^{\mathrm{u}}\left(1-\mathrm{s}_{\mathrm{E}}\right)+\left[\mathrm{r}_{\mathrm{Ek}}^{\mathrm{u}}\left(1-\mathrm{s}_{\mathrm{E}}\right)-\mathrm{r}_{\mathrm{Fk}}\left(1-\mathrm{s}_{\mathrm{Fk}}\right)\right]\left(1-\frac{\mathrm{TS} / \mathrm{Fk}}{\mathrm{r}_{\mathrm{Fk}}\left(1-\mathrm{s}_{\mathrm{Fk}}\right)}\right) \frac{\mathrm{Fk}}{\mathrm{Ek}}$

17 Siehe Miles/Ezzell (1980, 719) und Schultze (2004, 779ff., 789). Die Nachsteuer-Kapitalkosten werden hier durch die Multiplikation der Vorsteuer-Kapitalkosten mit dem Faktor $(1-\mathrm{s})$ ermittelt $(\mathrm{s}=$ Steuersatz). Zu möglichen Problemen einer solchen Definition vgl. Kruschwitz/Löffler (2004), dagegen a.A. Wilhelm (2005). 
Daraus resultiert:

$$
\mathrm{r}_{\mathrm{Ek}}^{\ell *}=\mathrm{r}_{\mathrm{Ek}}^{\ell}\left(1-\mathrm{f} \times \mathrm{s}_{\mathrm{Kap}, \mathrm{B}}\right)\left(1-\mathrm{x}_{\mathrm{B}} \times \mathrm{s}_{\mathrm{ESt}, \mathrm{B}}\right)=\mathrm{r}_{\mathrm{Ek}}^{\mathrm{u}^{*}}+\left[\mathrm{r}_{\mathrm{Ek}}^{\mathrm{u}^{*}}-\mathrm{r}_{\mathrm{Fk}}^{*}\right]\left(1-\frac{\mathrm{r}_{\mathrm{Fk}} \times \mathrm{s}^{*}}{\mathrm{r}_{\mathrm{Fk}}^{*}}\right) \frac{\mathrm{Fk}}{\mathrm{Ek}}
$$

\section{Zusammenfassung}

Für das Tax Shield bei einer DCF-Bewertung wurden in dem Beitrag Bewertungsformeln abgeleitet, die die Besonderheiten des schweizerischen Steuerrechts (Bundes- und Kantonssteuern) berücksichtigen. Folgende Einflussfaktoren wurden hierbei identifiziert:

Zunächst ist das Steuerniveau im Kanton der Kapitalgesellschaft (Bewertungsobjekt) zu berücksichtigen. Mit steigendem Steuersatz nimmt auch das Tax Shield zu. Grund hierfür ist, dass der Steuervorteil aus der Fremdfinanzierung einer Kapitalgesellschaft bei steigendem Körperschaftsteuersatz ebenfalls zunimmt. Allerdings ermittelt sich dieser Steuervorteil für Zwecke der kantonalen Steuer nicht aus einer schlichten Multiplikation des jeweiligen Steuersatzes mit den Zinsaufwendungen, die die Bemessungsgrundlage mindern. Vielmehr besteht eine Wechselwirkung mit den Bundessteuern (Abzugsfähigkeit der Steuern von ihrer eigenen Bemessungsgrundlage), die ebenfalls zu berücksichtigen ist.

Ferner wird das Tax Shield durch das Steuerniveau in den Kantonen des Kapitalgebers (Gesellschafter oder fremder Dritter) beeinflusst. Ob dies sich positiv oder negativ auf das Tax Shield auswirkt, hängt davon ab, ob Eigen- oder Fremdfinanzierung vorliegt: Eine hohe Steuerbelastung des Fremdkapitalgebers reduziert den Steuervorteil der Fremdfinanzierung, da die Fremdkapitalvergütungen einer entsprechend höheren Besteuerung unterliegen. Eine hohe Steuerbelastung des Eigenkapitalgebers erhöht hingegen den relativen Steuervorteil der Fremdfinanzierung.

Ein weiterer Gesichtspunkt ist die Ausgestaltung der Dividendenbesteuerung. Werden Gewinnausschüttungen im Sitzkanton des Eigenkapitalgebers/Dividendenbeziehers nur anteilig besteuert, wirkt sich dies mindernd auf das Tax Shield aus; dies gilt sowohl für Kapitalgesellschaften als auch für natürliche Personen, da diese durch den Beteiligungsabzug nahezu gleich gestellt sind. In Bezug auf eine Fremdkapitalgeber-Gesellschaft erhöht sich durch diese privilegierte Dividendenbesteuerung hingegen der Vorteil einer Fremdfinanzierung.

Hieraus ergibt sich, dass ein hohes Tax Shield vor allem dann entsteht, wenn im Kanton der zu bewertenden Kapitalgesellschaft und dem ihrer Anteilseigner ein hohes Steuerniveau herrscht, die Steuerbelastung im Kanton des Fremdkapitalgebers jedoch relativ gering ist.

Insgesamt bleibt festzuhalten, dass das Tax Shield insbesondere im Umfeld des schweizerischen Steuerrechts keinesfalls durch Multiplikation der Zinsaufwendungen mit dem Steuersatz im Kanton des Bewertungsobjekts ermittelt werden kann. Die kantonale bzw. kommunale Ausgestaltung der Steuerrechtsordnungen aller berührten Kantone muss in das Finanzierungskalkül Eingang finden. 


\title{
Symbolverzeichnis
}

\author{
BFCF Brutto-Free Cashflow \\ Ek Marktwert des Eigenkapitals \\ f Prozentualer Anteil der Besteuerung mit Körperschaftsteuer im Kanton des Kapitalgebers \\ Fk Marktwert des Fremdkapitals \\ G Reingewinn vor Zinsen und Steuern \\ Gk Marktwert des Gesamtkapitals \\ $\mathrm{Gk}_{0}^{1} \quad$ Marktwert des Gesamtkapitals eines verschuldeten Unternehmens \\ ICF Investitions-Cashflow \\ $1 \quad$ verschuldet (Index) \\ OCF Operativer Cashflow \\ $\mathrm{r}_{\mathrm{EK}}^{1} \quad$ Eigenkapitalkosten vor pers. Steuern eines verschuldeten Unternehmens \\ $\mathrm{r}_{\mathrm{Ek}}^{1^{*}} \quad$ Eigenkapitalkosten nach pers. Steuern eines verschuldeten Unternehmens \\ $\mathrm{r}_{\mathrm{Ek}}^{\mathrm{u}} \quad$ Eigenkapitalkosten vor pers. Steuern eines unverschuldeten Unternehmens \\ $\mathrm{r}_{\mathrm{EK}}^{\mathrm{u}^{*}} \quad$ Eigenkapitalkosten nach pers. Steuern eines unverschuldeten Unternehmens \\ $\mathrm{r}_{\mathrm{Fk}} \quad$ Fremdkapitalkosten vor pers. Steuern \\ $\mathrm{r}_{\mathrm{FK}}^{*} \quad$ Fremdkapitalkosten nach pers. Steuern \\ $\mathrm{r}_{\text {WACC }}^{1^{*}} \quad$ Gewichtete Kapitalkosten nach Steuern eines verschuldeten Unternehmens \\ $\mathrm{S}^{*} \quad$ Prozentsatz des Tax Shields \\ $\mathrm{S}_{\mathrm{E}} \quad$ persönlicher Steuersatz für Eigenkapitaleinkommen \\ $\mathrm{S}_{\mathrm{EK}}^{\text {natP }} \quad$ Gesamtsteuersatz auf Unternehmens- und Anteilseignerebene bei natürlicher Person als Eigenkapitalgeber \\ $\mathrm{S}_{\mathrm{EK}}^{\mathrm{Kap}} \quad$ Gesamtsteuersatz auf Unternehmens- und Anteilseignerebene bei Kapitalgesellschaft als Eigenkapitalgeber \\ $\mathrm{S}_{\mathrm{ESt}}^{\mathrm{BSt}} \quad$ Einkommensteuersatz auf Bundesebene $(11,5 \%)$ \\ $\mathrm{s}_{\mathrm{ESt}, \mathrm{A}}^{\mathrm{kkSt}} \quad$ Einkommensteuersatz auf kantonaler/kommunaler Ebene im Kanton A \\ $\mathrm{S}_{\text {ESt,B }} \quad$ Gesamtsteuersatz der Einkommensteuer im Kanton B \\ $\mathrm{S}_{\mathrm{ESt}, \mathrm{B}}^{\mathrm{kkSt}} \quad$ Einkommensteuersatz auf kantonaler/kommunaler Ebene im Kanton B \\ $\mathrm{S}_{\text {ESt,C }}$ Gesamtsteuersatz der Einkommensteuer im Kanton C \\ $\mathrm{S}_{\mathrm{ESt}, \mathrm{C}}^{\mathrm{kkSt}} \quad$ Einkommensteuersatz auf kantonaler/kommunaler Ebene im Kanton C
}


$\mathrm{S}_{\mathrm{Fk}} \quad$ pers. Steuersatz für Fremdkapital-Einkommen

$S_{F K}^{n a t P} \quad$ Steuerlast auf Zinseinkünfte bei natürlicher Person als Fremdkapitalgeber

$s_{F K}^{n a t P, C} \quad$ Steuersatz auf Fremdkapitaleinkünfte einer natürlichen Person im Kanton C

$S_{F K}^{K a p, C} \quad$ Steuerlast auf Fremdkapitaleinkünfte einer Kapitalgesellschaft im Kanton C

$\mathrm{s}_{\mathrm{Kap}}^{\mathrm{BSt}} \quad$ Bundessteuersatz für Kapitalgesellschaften (proportional 8,5\%)

$\mathbf{S}_{\text {Kap,A }} \quad$ Effektivsteuersatz auf Ebene der Kapitalgesellschaft im Kanton A

$\mathrm{S}_{\mathrm{Kap}, \mathrm{A}}^{\mathrm{kkSt}} \quad$ Steuersatz für Kapitalgesellschaften auf kantonaler/kommunaler Ebene im Kanton A

$\mathrm{S}_{\text {Kap,B }} \quad$ Effektivsteuersatz auf Ebene der Kapitalgesellschaft im Kanton B

$\mathrm{S}_{\mathrm{Kap}, \mathrm{B}}^{\mathrm{kkSt}} \quad$ Effektivsteuersatz für Kapitalgesellschaften auf kantonaler/kommunaler Ebene im Kanton B

$\mathrm{S}_{\text {Kap,C }} \quad$ Effektivsteuersatz auf Ebene der Kapitalgesellschaft im Kanton C

$\mathrm{S}_{\text {Kap,C }}^{\mathrm{kkSt}} \quad$ Steuersatz für Kapitalgesellschaften auf kantonaler/kommunaler Ebene im Kanton C

$S_{p, C} \quad$ Steuersatz auf Fremdkapitalzinsen

$\mathrm{T}^{\mathrm{u}} \quad$ Gesamte Steuerbelastung der Eigentümer eines unverschuldeten Unternehmens

TS Tax Shield

u unverschuldet (Index)

$\mathrm{X}_{\mathrm{B}} \quad$ Prozentsatz der Besteuerung von Dividendeneinkünften im Kanton B

$\mathrm{X}_{\mathrm{C}} \quad$ Prozentsatz der Besteuerung von Dividendeneinkünften im Kanton C

$\mathrm{X}_{\mathrm{GV}, \mathrm{B}} \quad$ Prozentsatz der Besteuerung im Rahmen der Freistellung von Dividendeneinkünften bei sich im Geschäftsvermögen befindlichen Gesellschaftsanteilen im Kanton B (50\%)

$\mathrm{X}_{\mathrm{PV}} \quad$ Prozentsatz der Besteuerung im Rahmen der Freistellung von Dividendeneinkünften bei sich im Privatvermögen befindlichen Gesellschaftsanteilen im Kanton B $(60 \%)$

$\mathrm{X}_{\mathrm{SB}, \mathrm{B}} \quad$ Prozentsatz der Besteuerung im Rahmen der Freistellung von Dividendeneinkünften bei sich in Streubesitz befindlichen Gesellschaftsanteilen im Kanton B $(100 \%)$

$Y_{E K, l} \quad$ Eigenkapitaleinkommen aus einer verschuldeten Unternehmung

$\mathrm{Y}_{\mathrm{EK}, \mathrm{u}} \quad$ Eigenkapitaleinkommen aus einer unverschuldeten Unternehmung

$\mathrm{Y}_{\mathrm{FK}} \quad$ Fremdkapitaleinkommen

$\mathrm{Y}_{\mathrm{GK}, 1} \quad$ Gesamteinkommen aus einer verschuldeten Unternehmung (Eigen- und Fremdkapital)

Z Zinsaufwand 


\section{Literaturhinweise}

Auge-Dickhut, S./Moser, U./Widmann, B. (2000): Die geplante Reform der Unternehmensbesteuerung - Einfluss auf die Berechnung und die Höhe des Werts von Unternehmen, in: Finanz Betrieb, Jg. 2, Nr. 6, S. 362-371.

Bachmann, C./Schultze, W. (2008): Unternehmensteuerreform 2008 und Unternehmensbewertung: Auswirkungen auf den Steuervorteil der Fremdfinanzierung von Kapitalgesellschaften, in: Die Betriebswirtschaft, Jg. 68, Nr. 1, S. 9-34.

Baetge, J./Niemeyer, K./Kümmel, J. (2001): Discounted Cashflow-Verfahren mit Beispiel, in: Peemöller, $V$. (Hrsg.): Handbuch der Unternehmensbewertung, Berlin, S. 263-360.

Ballwieser, W./Kruschwitz, L./Löffler, A. (2007): Einkommensteuer und Unternehmensbewertung Probleme mit der Steuerreform 2008, in: Die Wirtschaftsprüfung, Jg. 6, Nr. 18, S. 765-769.

Blumenstein, E./Locher, P. (2002): System des schweizerischen Steuerrechts, 6. Aufl., Zürich.

Berk, J./DeMarzo, P. (2007): Corporate Finance, 2. Aufl., Boston.

Brealey, R. A./Myers, S.C./Allen, F. (2008): Principles of Corporate Finance, 9. Aufl., New York u. a.

Dinstuhl, V. (2002): Discounted Cash-flow-Methoden im Halbeinkünfteverfahren, in: Finanz Betrieb, Jg. 4, Nr. 2, S. 79-90.

Drukarczyk, J. (2003): Unternehmensbewertung, 4. Aufl., München.

Drukarczyk, J./Lobe, S. (2002a): Discounted Cash Flow-Methoden und Halbeinkünfteverfahren, in: Achleitner, A.-K./Thoma, G. F. (Hrsg.): Handbuch Corporate Finance, 2. Aufl., Köln, 2. Ergänzungslieferung 2002, S. 1-32.

Drukarczyk, J./Lobe, S. (2002b): Unternehmensbewertung und Halbeinkünfteverfahren - Probleme individueller und marktorientierter Bewertung steuerlicher Vorteile, in: Betriebs Berater, Beilage 6 zu Nr. 38, S. 2-9.

ESTV (2008): Eidgenössische Steuerverwaltung: Steuerbelastung in der Schweiz, Kantonshauptorte Kantonsziffern 2008, Bern 2009. Abrufbar im Internet unter http://www.bfs.admin.ch/bfs/portal/ de/index/themen/18/22/publ.Document.122214.pdf, Stand: 02.03.2010.

Husmann, S./Kruschwitz, L./Löffler, A. (2002a): Tilgungseffekt und Kapitalherabsetzung, in: Die Betriebswirtschaft, Jg. 62, Nr. 5, S. 559-561.

Husmann, S./Kruschwitz, L./Löffler, A. (2002b): Unternehmensbewertung unter deutschen Steuern, in: Die Betriebswirtschaft, Jg. 62, Nr. 1, S. 24-42.

Kruschwitz, L./Löffler, A. (2004): Bemerkungen über Kapitalkosten vor und nach Steuern, in: Zeitschrift für Betriebswirtschaft, Jg. 74, Nr. 12, S. 1175-1190.

Kolb, A. (2008): Übersicht über die schweizerische Unternehmenssteuerreform II, in: Internationale Wirtschafts-Briefe, Gruppe 2, Fach 5, S. $361-368$.

Kubaile, H. (2006): Die Schweiz zählt in Europa weiterhin zu den ersten Adressen, in: Praxis Internationale Steuerberatung, o. Jg., Nr. 4, S. 96-102.

Laitenberger, J. (2002): Tilgungseffekt und Kapitalherabsetzung, in: Die Betriebswirtschaft, Jg. 62, Nr. 5, S. 555-559.

Laitenberger, J. (2003): Kapitalkosten, Finanzierungsprämissen und Einkommensteuer, in: Zeitschrift für Betriebswirtschaft, Jg. 73, Nr. 11, S. 1221-1239.

Lobe, S. (2001): Marktbewertung des Steuervorteils der Fremdfinanzierung und Unternehmensbewertung, in: Finanz Betrieb, Jg. 3, Nr. 12, S. 645-652.

Miles, J.A./Ezzel, J.R. (1980): The Weighted Average Cost of Capital, Perfect Capital Markets and Project Life: A Clarification, in: Journal of Financial and Quantitative Analysis, Jg. 15, Nr. 2, S. 719-730.

Schüler, A. (2000): Unternehmensbewertung und Halbeinkünfteverfahren, in: Deutsches Steuerrecht, Jg. 38, Nr. 36, S. 1531-1536. 
Schultze, W. (2003): Methoden der Unternehmensbewertung - Gemeinsamkeiten, Unterschiede, Perspektiven, 2. Aufl., Düsseldorf.

Schultze, W. (2004): Valuation, Tax Shields and the Cost-of-Capital with Personal Taxes: A Framework for Incorporating Taxes, in: International Journal of Theoretical and Applied Finance, Jg. 7, Nr. 6, S. 769-804.

Schultze, W. (2005): Unternehmensbewertung und Halbeinkünfteverfahren: Steuervorteile aus der Finanzierung deutscher Kapitalgesellschaften, in: Die Betriebswirtschaft, Jg. 65, Nr. 3, S. 237-257.

Schultze, W./Meyer, M.A. (2005): Die Kapitalflussrechnung in der Unternehmensbewertung: Bewertungskalkül und Gestaltungshinweise, in: Zeitschrift für Planung und Unternehmenssteuerung, Jg. 16, Nr. 3, S. 289-324.

SSK (2008a): Schweizer Steuerkonferenz, Die Besteuerung der juristischen Personen, Bern. Abrufbar im Internet unter http://www.estv.admin.ch/dokumentation/00079/00080/00736/index.html? lang=de, Stand: 02.03.2010.

SSK (2008b): Schweizer Steuerkonferenz, Die Vermögenssteuer natürlicher Personen, Bern. Abrufbar im Internet unter http://www.estv.admin.ch/dokumentation/00079/00080/00736/index.html?lang=de, Stand: 02.03.2010.

SSK (2009): Schweizer Steuerkonferenz, Unternehmenssteuerreform II, Bern. Abrufbar im Internet unter http://www.estv.admin.ch/dokumentation/00079/00080/00736/index.html?lang=de, Stand: 02.03.2010.

Wallmeier, M. (1999): Kapitalkosten und Finanzierungsprämissen, in: Zeitschrift für Betriebswirtschaft, Jg. 69, Nr. 12, S. 1473-1490.

Weigell, J. (2008): in: Debatin, H./Wassermeyer, F. (Hrsg.): Doppelbesteuerung, München, Band. 5, Anhang Schweiz.

Wilhelm, J. (2005): Bemerkungen über Kapitalkosten vor und nach Steuern - Anmerkungen zu dem gleichnamigen Beitrag von Kruschwitz und Löffler, in: Zeitschrift für Betriebswirtschaft, Jg. 75, Nr. 10, S. 1005-1012.

Carmen Bachmann, Dr., Dipl.-Kauffrau, ist wissenschaftliche Assistentin am Lehrstuhl für Betriebswirtschaftliche Steuerlehre der Universität Augsburg.

Anschrift: Universität Augsburg, Lehrstuhl für Betriebswirtschaftliche Steuerlehre, Universitätsstr. 16, D-86159 Augsburg, Tel.: +049 (0)821/598-4032, Fax: +49 (0)821/598-4214, E-Mail: carmen.bachmann@wiwi.uni-augsburg.de

Wolfgang Schultze, Dr., ist Professor für Wirtschaftsprüfung und Controlling, Universität Augsburg.

Anschrift: Universität Augsburg, Lehrstuhl für Wirtschaftsprüfung und Controlling, Universitätsstr. 16, D-86159 Augsburg, Tel.: +049 (0)821/598-4130, Fax: +49 (0)821/598-4224, E-Mail: wolfgang.schultze@wiwi.uni-augsburg.de

Die Autoren danken den anonymen Gutachtern für wertvolle Hinweise. 